\title{
Analogies of genetic and chemical code. Supplement 1
}

\author{
Miloje M. Rakočević \\ University of Niš, Faculty of Sciences and Mathematics, Department of Chemistry, \\ Višegradska 33, 18000 Niš, Serbia
}

\begin{abstract}
In the original work, for which this is a Supplement, I presented analogies of the genetic and chemical code (Rakočević 2018b, in relation to the source work from 1991). (Further: instead of "Rakočević" I use the abbreviation "MMR".) There I gave three Tables of the Periodic System of the Elements (PSE) in which I dealt with the problem of stable and unstable elements; in the sense that an unstable element is one that possesses at least one primordially unstable isotope, while elements that do not possess such isotopes are stable. I have shown that for the number of stable and the number of unstable elements there are strict regularities and a strict law; the same law that is valid for the association of codons to more complex and to less complex amino acids in the genetic code. I have not dealt with the question of the number of stable and the number of unstable isotopes, what I do now in this Supplement. [An expanded version of the original paper whose Supplement this is: OSF Preprint DOI 10.31219/osf.io/mxecj]
\end{abstract}

Keywords: Chemical code, Genetic code, Periodic system of chemical elements, Protein amino acids, Lanthanides, Periodic system of numbers.

Of the three PSE Tables I gave in a previous original paper (MMR, 2018b), for which this is a Supplement, only one contains the number of natural isotopes. It is a Table with six columns, in an arrangement of 1:2:3, in the form of monads-diads-triads, with one, two and three columns, respectively (Table 2 in: MMR, 2018b, p. 295). ${ }^{1}$ In this Supplement, however, for elements up to the limit of stability/instability (up to Polonium, inclusive it) (Table 1) the number of both, stable and unstable isotopes is indicated: in front of the sign " + " the number of stable, and after the sign is given number of unstable isotopes (corresponding to the notation in: MMR, 2018b, Survey 1, p. 294). Only stable isotopes in stable elements are marked with color, which we are dealing with in this research: red in diads, blue in triads and green in monads; the same colors are also used to mark the groups to which the elements belong in the classical PSE Table with short and/or long periods (with groups, in total: I to XIV).

\footnotetext{
1 “This six groups PSE one can compare with the first Mendeleev's Table of PSE, with 6 groups, March 13, 1869, in: (Kedrov, 1977, p. 128-129, photocopy II); also in: (Mendeleev, 1869, reprinted in 1970)" (MMR, 2018b, p. 294). [Additional note: The first Table of Mendeleev was with 6 columns, as given in the cited references, and the last Table with 14 columns, as explained in the Preliminaries of MMR, 2018b. This cannot be without being indicative from several aspects, especially from the aspect of questioning for the quantities "6" and "14", for their chemical meaning. As far as I am concerned, if it were not so with Mendeleev, there would be neither this work of mine nor the works from 2018-2020 on the PSE with 6 groups and 14 columns and vice versa, listed in References.] (Cf. Footnote 10)
} 
It makes no sense to deal with determining the number of isotopes in unstable elements (either stable or unstable isotopes), at least for now, because it is more difficult experimentally to determine the demarcation limit of stability/instability in the isotopy of unstable elements. [A good example is the dilemma that IUPAC had, regarding Europium - whether it is stable or unstable - which I discussed in the previous paper (MMR, 2018b, Footnote 8, p. 296) (Cf. Box 1).] The reason more for this impossibility could be even deeper; the same one that S. Hawking and L. Mlodinow state - Heisenberg's Uncertainty principle (Hawking, Mlodinow, 2005, Chapter 11 ${ }^{\text {th }}$, the third standpoint on the possibility / impossibility of the existence of a unified theory of the Universe). However, these are questions for future researches, and for the current present only the question of determining the trend of agreement of experimental results and models presented in the original paper whose Supplement is this, as well as of the findings we present in this Supplement is important.

As can be seen from Table 1, the number of stable isotopes in stable elements, in diads-triads-monads (in that order) is such a number that it does not indicate chaoticity ${ }^{2}$ but strict regularity: it corresponds to a series of natural numbers "20-30-50-80-130", which (series) strictly corresponds to the Fibonacci sequence "2-3-5-8-13". The fact that regularities are in the question, is also indicated by the sum of number which denote groups in the Table of short and/or long periods, in the positions of stable elements (Table 2).

The arithmetical arrangement given in Table 2 is generated as follows. First, the nine "ordinary" decades of the decimal number system are added to the nine corresponding modular ones: the first to the first, the second to the second, and so on. [Here the number 19 appears to be a specific "threshold of modularity" (Table 2, second column)] The members of the set of "ordinary" decades are then added to the obtained sums again, but in the reverse order. Then two more operations are performed, as described in the legend of Table 2. The final result is the last column with three subcolumns. The first result in that column is: $199-90=109$. And that is the result we get as the final result of adding the "number of groups" in monads, diads and triads of Table 1: the 90 for monads and diads, and 109 for triads, which in total is 199.

All these results follow, provided that the PSE contains 14 groups and not 18 (as we said); that the lanthanides are distributed in each group per one, and not all 14 in the third group; that hydrogen is not in the first but only in the seventh group, as a neighbor of helium which is in the eighth group; as well as that noble gases in the calculation of the sum of the number of groups are taken as members of the eighth group, although they are

\footnotetext{
${ }^{2}$ Chaoticity would inevitably have to occur if PSE has 18 groups, as it is in "current science". In other words, the appearance of such strict regularities confirms the accuracy of all three of our PSE Tables. Confirmation more is the distribution of stable isotopes in stable elements within the classical PSE arrangement (with 14 groups) as we will show in Table 3.
} 
at the same time a zeroth group, from the aspect of their non-reactivity (cf. Footnote 6). The result for the "group number" as we have seen is such that it strictly corresponds to a specifically generated arrangement from a series of natural numbers (Table 2). ${ }^{3}$ On the other hand, the fact that the "chemical code" 4 , through the sum of the number of groups in the PSE, holds a connection with the series of natural numbers, speaks us of a possible unity, and not only of an analogy of these two main natural codes. ${ }^{5}$

Box 1. The excerpt from: MMR, 2018b, p. 296

\subsection{Experimental limits and an obvious trend}

Having in mind the fact that the stability/instability of isotopes is experimentally established, it must be said that the presented 100 -percent-agreement between the real state (number of stable and primordially unstable isotopes) and the models, presented in Survey $2 \mathrm{a}$ and $3 \mathrm{a}$ (in relation to Survey $3 \mathrm{~b}$ ), must be taken (and considered) only as a trend, which should serve for further researches in both directions: for checking experimental results, and for checking the agreement of experimental results with the theoretical models.

In support of the mentioned above, there are some examples in the current state of affairs (which may possibly spoil the above 100-percent-agreement). In relevant literature one can see that potentially three of ten isotopes of tin are radioactive, but have not been observed to decay. One of these three actually has been identified as Sn-124, and in IUPAC sources is indicated its half-life decay a little larger than $1 \times 10^{17}$ years. In addition, one of the seven (stable) isotopes of Gadolinium (Gd-152) we take to be unstable with a half-life decay of $1.08 \times 10^{14}$ years, while the isotope (Gd-160) with a half-life decay larger than $1.3 \times 10^{21}$ remained in a stable status. Overall, we marked the isotopic state of Gadolinium $(6+1)$. These are only two our interventions in relation to the IUPAC document which are, as follows from the above, also consistent with the understanding of isotopy in current science. $^{8}$

\footnotetext{
${ }^{3}$ The testing the correspondence of the ordinal number of chemical elements in PSE with series of natural numbers in the manner as in Table 2 does not make sense, because any result to be obtained would inevitably be less obvious than immediate obviousness: one hundred percent correspondence of the ordinal number of chemical elements with the series of natural numbers stems from the fact that the atom of the first element (hydrogen) contains one proton, the second two, the third three and so on.

4 "A natural code is such an essence that can be represented by an appropriate mathematical expression, which contains a sequence from the series of natural numbers within itself. From this follows that both are natural codes, the Genetic code as well as the Periodic system of chemical elements (PSE)" (MMR, 2017b, in Abstract).

5 "In determination of the genetic code, except two inherent alphabets - 20 amino acids and four amino bases (two pyrimidines \& two purines) - is involved still one 'hidden alphabet', a series of natural numbers, with all its regularities and laws" (MMR, 2011a, p.4).
} 
Additional Note to Box 1 . In connection with the position set out in the first paragraph ,that the presented 100-percent-agreement between the real state ... and the models ... must be taken (and considered) only as a trend ...", I want to add one clarification now. It is not just a trend only, but an unexpected agreement of experimental results with an unexpected model. [Ideal agreement and ideal model, as a reference system for all future experimental and theoretical researches, in this scientific area.] Why the unexpected? What else to say about the result (concerning the number of stable isotopes in stable elements): 20-30-50-80-130 in the Periodic Table with 6 columns (Table 1), and the result 62-64-66-68 in the Periodic Table with 14 columns (Table 3)? In the first case, the numerical notation of the obtained quantities is such that in all cases the final digit is zero, and the first number in notation is a Fibonacci number, such number which follows from the continuous Fibonacci sequence; in the second case, the sixth quartet sequence from a series of even numbers was obtained, corresponding to the combined fourth and fifth sequences previously found in GC (48-50-52-54). [The sum of the numbers in the genetic-coding sequence 48-50-5254 is 204 , which corresponds to the number of atoms in the 20 side chains of 20 protein amino acids, and the sum of the numbers in the chemical-coding sequence 62-64-66-68 is 260, which is twice the number of stable isotopes in stable poly-isotopic elements. (Relationships among two sequences are presented in Surveys $1-3$ in correspondences with Surveys 4-8.)]

It is almost immediately obvious that there is no causality here, and yet there are strict regularities; such regularities as I have presented in the previous three papers (MMR, 2018a, $2018 \mathrm{~b}, 2019 \mathrm{a}$ ), and in this paper. In other words, it is about the essence of the chemical and genetic code, which is different from the understanding of natural systems in current science, which was first pointed out by V. Shcherbak (1993, 1994), and later by many other researchers. ["So, the type of new arithmetical regularities combines both sufficient freedom for choosing and using any biologically important molecules and severe restrictions on chances being involved in this proces. The physical nature of such a phenomenon is so far not clear" (Shcherbak, 1993, p. 401).]

I dare to present a hypothesis (for further researches) according to which this is an indirect causality, whose scenarios are currently difficult to enter and decipher. However, it may make sense to continue presenting new regularities of the same (or similar) type, with the idea that it will then be easier to find an answer as to what the essence is.

In the next research step, we calculate the number of stable isotopes in the classical PSE Table (14 groups and 6 rows to the limit of stability, to Polonium), according to the distribution of elements as given in the original paper (MMR, 2018b, Survey 1, p. 294). This new result is presented in Table 3. The number of isotopes in the upper and lower areas, then on the first diagonal (red) and the second diagonal (blue) is such that it corresponds to the sequence of even numbers: $62,64,66,68$.

We have, thus, a sequence from the series of natural numbers $(62,64,66,68)$ as a result in the Periodic Table with 14 columns, for the number of stable isotopes in stable poly-isotope elements (Table 3); and also the sequence (20-30-50-80-130), strictly corresponding to a sequence of Fibonacci numbers, as a result in the Periodic Table with 6 columns (Table 1). The first sequence is obtained only when the columns (groups of chemical elements) are distributed according to the logic of Boolean spaces ${ }^{6}$; the second

\footnotetext{
6 "In a previous work (MMR, 1991) we proposed a hypothesis that the PSE of the short period groups corresponds to the Boolean cube as well as the PSE of the long period groups corresponds to the Boolean
} 
was obtained only under the condition that 6 columns were split into monads, diads and triads, with 1, 2 and 3 columns, respectively.

When asked why this is so, we have no answer, but we can draw an analogy with the genetic code. This is first discussed above - also a quartet sequence - the number of atoms in 20 side chains for 20 protein AAs (48-50-52-54); and then the determination of GC by the Golden mean, which is, indirectly, the determination by the Fibonacci sequence (Remarks 1 and 2).

Remark 1. The numbers (vertices) in $\mathrm{n}$-dimensional Boolean cube, $\mathrm{B}^{\mathrm{n}}\left(\mathrm{B}^{\wedge} \mathrm{n}\right)$, are divided into lower and higher rank numbers as follows. In lower-ranking spaces, the numbers are in this order: 0-4, 1-5 (000-100 and 001-101), and so on. In higher-ranking spaces, the numbers are in this order: $2-6,3-7$ (010-110 and 011-111, and so on. In Boolean cube $\mathrm{B}^{\wedge} 3$ on "below" are the first four numbers from a series of even natural numbers $(0,2,4,6)$, while "above" are the first four numbers from a series of odd natural numbers $(1,3,5,7)$.

Remark 2. The four-member sequence of even natural numbers in genetic code follows from (MMR, 2011b: Table 9 in relation to Table 10 and Figure 5 in relation to Figure 6). The said Table 9 is here (in this paper) Table A1, and Table 10 is here Table A2. ${ }^{7}$

$*$

The system-arrangement in Table 3 consists of two systems, the first (above) and the second (below). The first system starts with a lower rank subsystem (L) and continues with a higher rank subsystem $(\mathrm{H})$. In the second system (below) it is the other way around. In the upper area there are $7+11=18$ of stable chemical elements, and in the lower area $6+12=18$. The group number above is $27+62=89$ and below $30+80=110$. The number of stable elements in odd groups is: $7+6=13$, while the number of stable elements in even groups is $11+12=23$; exactly as stated in the original paper (MMR, 2018 b, Survey 3a, p. 296); and exactly (13-23) as we find in the starting position of a specific arithmetic-chemical system contained in the Genetic Code (MMR, 2020, Table 2, p. 14/6).]

By comparing Table 1 (PSE with 6 columns) and Table 3 (PSE with 14 columns) we see that the result of the distinction of the number of groups into two areas, from result 90/109 passed to result $89 / 110$; therefore with the smallest possible change by \pm 1 . [In search of the possible meaning of the choice of quantity $89 / 109$, we wonder whether there may be a correspondence with the properties of these numbers, presented in Tables B1 and B2.] By comparing these two tables (Table 1 and Table 3), we find an unusual

hypercube; the role of the $16^{\text {th }}$ group in such a case (in a cyclic ordering) plays either zeroth group or the first group "(MMR, 2018b, p. 292).

${ }^{7}$ The two tables and two figures we cite here concern the determination of the genetic code by the golden mean on the binary code tree. However, as this is a binary tree in direct correspondence with the Farey tree which is determined by the Fibonacci sequence, the analogy is complete (MMR, 1998, Figure 1 in relation to Figure 2). The manner how the four-membered sequence of even numbers (quartets) appears in the genetic code is shown here in Table A2 in relation to Table A1. 
inversion. In Table 1 we have that the number of isotopes in monads-diads-triads corresponds to the Fibonacci sequence, while the number of groups corresponds to the decades sequences of the decimal number system. In contrary, in Table 3 everything is the other way around: the number of isotopes does not correspond to Fibonacci sequences but to the quartets sequences of the decimal number system, and the group number corresponds to Fibonacci sequences in a specific way - via Fibonacci "triangles" (result $89 / 110$ in the last column of Table 3 compared with the same result in the third row of Table 4).

The arithmetical arrangement given in Table 4 is generated as follows. The necessary and sufficient condition for the first possible validity of the Fibonacci law is to have (in a series) the first two natural numbers. And the first two natural numbers in Boolean spaces are $(0,1)$, which are not numbers only in the arithmetic sense but are points in the space. ${ }^{8}$ By applying the Fibonacci law over them, the zeroth Fibonacci "triangle" (Fibonacci triplet) is obtained and all other as in the order indicated in Table 4. As we can see, in the third step of a specific logical square, we find the numbers (spatial points in Boolean space) that correspond to the "Group number" for LH (89) and HL (110) of PSE, as indicated in last column of Table 3. [Table 5 is one step further in relation to Table 4, and in Tables 6, 7 and 8 the details of the relationships in the last two columns of Table 5 are presented.]

The validity of the "Neighborhood law" (Tables 4 and 5) is necessary for the origin of these two results ( 89 and 100): the double value of the second member of any Fibonacci triangle is equal to the sum of the third member of that triangle and the third member of the previous one. [Examples: $3+3=6$ and $5+1=6$; or: $13+13=26$ and $21+5=26$. ] In order for the law to apply to the zeroth Fibonacci triangle, it is necessary the existence of the space of negative Fibonacci triangles (Table 5 in relation to Table 6).

In Tables 7 and 8 we find correspondence with 2-bit, 4-bit and 6-bit binary trees: the numbers marked in red represent one third (1/3) of the corresponding binary tree: $0-3$ for the 2-bit binary tree (with 4 one-word words); 0-15 for the 4-bit binary tree (with 16 twoletter words) and 0-63 for the 6-bit binary tree (with 64 three-letter words), as found in the genetic code. No other binary tree fits this regularity. [Note: One third of the binary tree represents a half of the harmonic mean of the whole of binary tree and its half.]

\footnotetext{
${ }^{8}$ Hence the unfounded criticism directed at many chemists, over the decades, that they allegedly practiced numerology. [Andrea I. Woody, Department of Philosophy, University of Washington, Seattle: "There were likely significant differences of opinion about the appropriateness of ... predictions, which could appear overly speculative and thus not properly empiricist. Indeed, reticence is not hard to imagine given the surface similarities between various codifications of periodicity in the 1860s and 1870s and numerical relations suggested in earlier decades that had been ridiculed as pieces of Pythagorean numerology. More generally, there were likely relevant differences in interests and intellectual style among the individuals involved and across the communities they inhabited" (Woody, 2014) (Cf. Footnote 10).]
} 
Once again, we return to the analysis of the relationship between the two sequences: sequences 62-64-66-68 in the chemical code and sequences 48-50-52-54 in the genetic code (Surveys 1-3). Given the fact that such four-member (quartet) sequences exist in natural codes, it makes sense to test the whole series of such sequences, starting from the first sequence (2-4-6-8). ${ }^{9}$ We are first interested in the demarcation line between that initial sequence and the sequence valid for the chemical code: 2-4-6-8 versus 62-6466-68 (Survey 1, in relation to Survey 3); in a parallel step, we analyze the relationship of two sequences: of chemical code and of genetic code. As we can see, the distance between the members of the two code sequences is 14; the distance between the first and the chemical code sequence is 60 ; the distance between the chemical code sequence and the last sequence in the set of two-digit numbers is 30 (Survey 2, in relation to Survey 3). Knowing that the key quantities that Mendeleev dealt with were actually these two quantities, 6 and 14 (Remark 3), we can conclude that it must have something to do with each other. ${ }^{10}$ (Cf. Survey 4 and Box 2)

Remark 3. The first Table Mendeleev made and distributed to the world's key chemists had 6 columns (Mendeleev, 1969, reprinted in 1970; Kedrov, 1977, p. 128: photocopy II), and the last one he compiled near the end of his life had 14 columns (cf. MMR, 2018b, in Preliminaries) (Footnote 1 in this paper). It must be admitted here that Mendeleev, in addition to his other predictions, also predicted that PSE must exist in form with 6 columns as well as with 14 columns. This is especially important today when we know that his allegedly erroneous calculations in the manuscript Table of short periods (recorded result 37 instead of 27 and 77 instead of 67) (Kedrov, 1977, p. 128, photocopy X) are not errors but dealing with quantities that will be much later detected in the genetic code (MMR, 2008: Tab. 3, p. 4; 2009: Table A.1, p. 21; 2017c: pp. 61-62 and footnote 34).

On the left side of Survey 4 we have the relationships among two sequences, the chemical code sequence (62-64-66-68), and the genetic code sequence (48-50-52-54). On the right side in relation to the first possible quartet sequence (2-4-6-8), two sequences are placed; the sequence (42-44-46-48) which refers to the number of atoms in 20 amino acid molecules, in their 20 "heads" (amino acid functional group: $20 \times 9=$ 180 ), and the sequence of the genetic code, that we have already considered so far (4850-52-54) and concerns the number of atoms in 20 amino acid molecules, this time in their "bodies", that is, their side chains (Remark 4).

\footnotetext{
${ }^{9}$ Of course, all the time we are talking about sequences of even numbers within a series of natural numbers. When it comes to GC, we know that this sequence has a real meaning: it is a pattern in which 20 AAs are splitting down into four types of diversity (MMR, 2011b, Figure 2, p. 822).

10 Andrea I. Woody, Department of Philosophy, University of Washington, Seattle: "In its nineteenth century formulation, the periodic law expresses the empirical generalization that trends in various chemical and physical properties of the elements tend to follow a single repeating pattern with respect to increasing atomic weight. According to historical lore, the validity of the law was demonstrated originally by a series of remarkable predictions made by the discoverer, the now immortal Dmitri Mendeleev.") (Woody, 2014) (Cf. Footnote 8).]
} 
Remark 4: Whenever a question is asked for a given number whether it can be displayed in the form of a quartet sequence, it should be divided by four, andt this implies an integer as the result of that division. In the case of 20 amino acid "heads" $(20 \times 9=180)$, dividing by 4 gives 45 . Therefore, the two inner members of the quartet sequence must be 44 and 46 , and the two outer 42 and 48. Up to the quartet sequence for the number of atoms in 20 side chains we came, however, by further research, starting from the results of V. Sukhodolets' (Table A1 in relation to Table A2).

\section{Box 2. Additional Explanation of Survey 4}

The number of monoisotopic elements is given at the very bottom on the left, which is at the same time the number of their stable isotopes (20). Data are also given for the number of groups in which the monoisotopic elements are found: for triads 42 instead of 44 because Beryllium is taken as if it were in the "zeroth" group (Box 2). Hence the "number of groups" of monoisotopic elements is 101 (Survey 11 in relation to Survey 13, in row "total"; together with the "number of groups" of poly-isotopic elements (199) equals 300 (Survey 13, last row), which quantity corresponds to the same quantity (300 as a specific difference) in GC (Table 9). On the right side (at the bottom) we have that the number of stable isotopes in poly-isotopic stable elements is as follows: in $(\mathrm{s}+\mathrm{p})$ elements $65-10$ and in $(\mathrm{d}+\mathrm{f})$ elements $65+10$, which is a kind of balance in relation to the arithmetic mean (Survey 13, first and second row, in red color). This change for \pm 10 in the number of isotopes corresponds to the change for \pm 11 in the "group number". In Survey 13, third row, in pink color there is 98 as $109-11$ and 101 as $90+11$; the result $90+109$ $=199$ we have on the top of Table 2, last column, in red color, and directly in Table 1 as the number of poly-isotopic elements, in the monads-diads-triads distinction: 90 in monads and dyads together, and 109 in triads.

But, even without monoisotopic elements, a quantity of 300 can be obtained if ( $s+p)$ elements are taken twice (101 + 101 in "group number", as it is done in Survey 4: down on the right). Analog such situations - with duplication of one and the same entity (its quantity) - we have found several times in GC (MMR, 2019b, 2020). Perhaps the best example is with duplication of AAs of non-alanine stereo-chemical types. Only in such a manner an extraordinary hierarchy and order of AAs is obtained from the aspect of the polarity of their molecules, as well as their biosynthetic precursors (Rakočević and Jokić, 1996, Survey 1 and Table 1).

\section{Box 3. Where did Beryllium in the "zeroth" group come from?}

In the process of calculating the "number of groups" of monoisotopic elements (Survey 4), in the sense - to add the numbers of all groups in which the monoisotopic elements are located - it is realized that Beryllium enters the account not with the number 2 but with the number 0 ? The answer to the question goes through the following insight. It is almost immediately obvious that PSE, from the aspect of reactivity-inertness, starts with a kind of a specific logical square, in the Gray Code order: $01 \mathrm{H} ; 00 \mathrm{He} ; 10 \mathrm{Li} ; 11 \mathrm{Be}$. In front of the state of non-reactivity, that is inertness (as $\mathrm{He}$ ), there is a chemically reactive element, $\mathrm{H}$; behind inertness also comes reactivity (as Li), and at the end point of the logical square - Beryllium, in a kind of "threshold of inertness".

After this insight, we compare this state with the state (and position) of inert elements (noble gases) in the arrangement given in Table 3. From the aspect of their non-reactivity they are, as we have already said, zeroth group, but in calculating the number of groups enter as the eighth group. The situation is reversed with Beryllium: despite being only on the threshold of inertness, and 
realistically is not in zeroth but in second group, it enters the sum of the number of groups with the number 0 (zero). Evidence in this regard is Table B6: If Be entered this arrangement with number 2, the result would be: 59/44, instead of 59/42 (59 as the number 14, and number 42 as number 6, according to module 9, respectively, as expected for PSE, in terms of number of groups / periods.

As a result of summation, on the right side of Survey 4 the following quantities are obtained: 204, number of atoms in 20 side chains of AAs (half: 102); 180, number of atoms in 20 "heads"; a total of 384 [which number is also found in Platon's Timaeus (Remark 5)] On the left side (below), a new sequence is given in two lines: 72-74-7678 , which also represents the chemical code sequence created by adding 20 monoisotopic elements to the already discussed sequence 62-64-66-68. The complete result for the number of isotopes (and group number) in the monoisotopic elements, that follows from Table 1, is given at the bottom of the Survey 4. ${ }^{11}$ The outcome for the total number of isotopes in all stable isotopes of all stable elements is 150 . The double value of that number (according to the sequence 72-74-76-78) is 300. However, this number also represents a single value of the sum of the number of PSE groups in which (groups ) stable elements are placed $(36+20=56) .{ }^{12}$ [From Surveys 9 and 10 follows "Group number" for poly-isotopic elements: $(41+60)+(16+82)=101+98=199$; from Surveys 11 and 12 follows "Group number" for monoisotopic elements: $34+67=101$; in total, "Group number" for all stable isotopes in all stable elements equals: $199+101=300$.]

Remark 5: Plato's arithmetical progression: within one of the sequences of the series $2^{\wedge} \mathrm{n}(\mathrm{n}=0$, $1,2,3, \ldots)$; that is, at the very beginning of the geometrical progression with quotient 2 , when their harmonic and arithmetic mean are inserted between numbers 1 and 2, and then the appropriate calculations are performed (MMR, 2011b, Tab. A.2, p. 839; cf. also Table B4 in this paper) (Platon, 1988, 1970)

\footnotetext{
11 Monoisotopic elements are considered as stable elements, with one stable isotope each, without primordially unstable isotopes. The only exception is Bismuth: "IUPAC Periodic Table of the Elements and Isotopes" (Google, 9.07.2020): "Radioactive isotope having a relatively long half-life $\left(2.0 \times 10^{19}\right.$ years $)$ and a characteristic terrestrial isotopic composition that contributes significantly and reproducibly to the determination of the standard atomic weight of the element in normal materials." From this statement of IUPAC, however, it follows that Bismuth is a stable monoisotopic element. As far as I am concerned here too I was in a situation to assess the significance of the experimental result in the same way as I did in the original work (MMR, 2018b) for Tin and Gadolinium (Box 1).

${ }^{12}$ The number 36 as the square of number 6 (the first perfect number) to be togethwr with number 56 as a double value of the number 28 (second perfect number). [Possible correspondence with the unique arithmetical arrangement is indicated in Table B3.] We found the same logic for the relationships of the number of atoms in nucleotide bases: [U 12, C 13, (14), A 15, G 16] versus the number of atoms in nucleotides: [UMP 34, CMP 35, (36), AMP 37, GMP 38] (MMR, 1997b, pp. 62-63; www.rakocevcode.rs), which all together point to the validity of the principles of similarity and self-similarity within the unity of two codes - chemical and genetic ones. (Cf. MMR, 1997a, in relation to Tab. 2 in MMR, 2019b; cf. also last column in Table 9 in this Supplement)
} 
The quantity of "300" for me has been a "missing link" within one specific "logical square" for years: [(0) 204, (1) 220, (2) 284, (3) 300] (Table A8). On one diagonal of that square is the first pair of friendly numbers (220-284), and on the other diagonal: 204 as the number of atoms within 20 amino acid side chains; and the number 300, as discussed herein, is the "group number" and twice the number of stable isotopes, and, finally, as the difference of the number of nucleons and the number of atoms in the GC (Table 9). ${ }^{13}$

Prediction. From the presented "logical square" [(0) 204, (1) 220, (2) 284, (3) 300] (Table A8) and from all other research results mentioned in this Supplement, and having in mind the fact that the entire universe is built of the same chemical elements, the presented "logical square" itself must be universal. Wherever in the universe there is a planet with the same physico-chemical conditions that exist on planet Earth, or near to being the same, there is the same kind of terrestrial life, or near to being the same.

Explanation of Prediction. Terrestrial life testifies to us that its origin, duration and survival are directly conditioned by the existence of the first possible non-metallic "island" of chemical elements in the Periodic Table, in an arrangement determined by the immediate neighborhood (by the said "Neighborhood island"). The first possible nonmetal in PSE, going from left to right (from metals to non-metals) is carbon, followed by neighbor to neighbor: nitrogen, next to it oxygen, in a diagonal neighborhood with hydrogen in the seventh group, and a vertical neighborhood with sulfur, analogous to the neighborhood of nitrogen to phosphorus.

In this Supplement, we have dealt only with the "Standard Genetic Code" in relation to the chemical code, to that part of it which refers to stable chemical elements. I have not dealt here with other genetic codes, of which there are quite a number today. This is because I have repeatedly pointed out in my works that these genetic codes represent only deviations from the standard, within the given degree of freedom. In a way, here we have, mutatis mutandis, an analogy with the chemical code, in the following sense: in the chemical code there are strict determinations and balances only for stable elements and their stable isotopes, and in the genetic code strict determinations and balances exist only within the standard genetic code, while all others have a high degree of freedom in

\footnotetext{
${ }^{13}$ The said three meanings of quantity "300", as the sum of the numbers of groups in PSE in which there are stable elements; as double values of number of stable isotopes; and as differences in the number of nucleons and the number of atoms (Table 9), leads us to the conclusion that Nature mixes here "chalk and cheese" ("lime and cheese"), and at the same time attributes to codons both the meaning of "material" (amino acid molecules) and the meaning of events (start and/or stop in protein biosynthesis). I have no other explanation for all this than the one with which I ended one of my previous papers, and it comes down to the stand point that these facts again "appealed to Aristotle and to his idea of unity of form and essence" (MMR, 2004a, p. 233]
} 
deviations and exceptions. This applies both to the assignment of codons to amino acids as well as to the expansion of the set of canonical amino acids within GC. ${ }^{14}$

In the case of codons assignment to amino acids, deviations from standard GC, in terms of exceptions are almost a well-known fact (MMR, 2018a, Box 2, p. 41). In the case of including of $21^{\text {st }}$ and $22^{\text {nd }}$ AAs, the problem is almost unresolved. As for me, in this regard, at least I gave a hint where "iacet lepus" (MMR, 2011b, Fig. 11, p. 835; here: Table A7) (Box 4). For the $21^{\text {st }}$ amino acid, Selenocysteine, the place can be found, in the area marked as "less variant" AAs, where Cysteine is already found (Table A7); and for the $22^{\text {nd }}$ amino acid, Pyrrolysine, it is the area of the "more variant" AAs, where Lysine is already located. In both cases, there is the space for possible other "variant" AAs, but in all such cases the balances we have presented here no longer apply: then there is a relatively high degree of freedom in deviations on the scene ${ }^{15}$.

Box 4. Explanation of Table A7 (MMR, 2011b, Section 4.3, p. 834)

"Now the question what is the meaning of the concepts (notions) of "invariant" or "variant". It is easy to understand why, for example, the glycine and alanine are invariant molecules; ... But, it is harder to understand why valine with a propyl group as its side chain is also invariant if there is (in the nature) simpler ethyl group.... There we see the sense, meaning and meaningfulness logic of selection: after the first possible case of non-hydrocarbonicity come possible cases of hydrocarbonicity; then the first possible case of openness, then of halfcyclicity, cyclicity and branching (hence the meaning of four stereochemical types of AAs). ... If, however, could be possible second, third etc. cases, then the invariant AAs outlined here would not be invariant. Hence it makes sense to talk not only about more or less variant, but also of more or less invariant (Table A7). The arrangement in Table A7 shows that the splitting of AAs into two classes - variant and invariant - is not only a formality ${ }^{16}$, or just a matter of chemical structure of molecules, but also the matter of amino acid functions (polarity). In column "more" (more variant and more invariant) there are six AAs, two polar (KR) and four semi-polar (GP and HW) ${ }^{17}$ In column "less" (less variant and less invariant)

\footnotetext{
14 Already the first discovered case of codon polysemy (AUA encodes isoleucine in the standard and methionine in the mitochondrial genetic code) was enough to see that in matters of the genetic code it is not "test tube chemistry" but "systemic chemistry" on the scene (MMR, 2019b, p. 3). A reason more for such an insight was the knowledge that codons can encode "something" molecular and "nothing" molecular ("stop" event, for example).

${ }^{15}$ I presented the basic essence of deviant genetic codes in one of the previous papers (MMR, 2018a, Box 2 , p. 41). However, that was not enough for the reviewer of my next paper, but he asked me to write again (MMR, 2020, Appendix). It turns out that whatever the author writes, he must rewrite in the next paper, because the reviewer does not have time to read what has been written before.

16 [Footnote 16 in source paper]: The matter of formality/ non-formality is also the atom number pattern: $(60+10), 66,(78-10)$ in relation to the pattern which follows from the golden mean determination: 60 , 66,78 as it is presented in (MMR, 1998)

${ }^{17}$ [Footnote 17 in source paper]: .... Lysine $(\mathrm{K})$ and arginine $(\mathrm{R})$ are polar in both parameters: hydropathy and cloister energy. On the other hand glycine $(\mathrm{G})$ and triptophan $(\mathrm{W})$ are polar in hydtropathy and nonpolar in cloister energy. The histidine $(\mathrm{H})$ is polar in hydropathy, but neutral in cloister energy. The $\mathrm{P}$ is special case: it is polar from the aspect of hydropathy, and non-polar from the aspect of polar requirement (Woese et al., 1966; Konopel'chenko and Rumer, 1975; Rakočević and Jokić, 1996, Survey 1).
} 
there are 14 AAs, seven polar and seven non-polar. If, however, do not look at the number of molecules than at the number of atomic associations then they are seven polar and six nonpolar. [The AAs leucine (L) and isoleucine (I) are one and the same atom association through the structural isomery.] Altogether, the amino acid molecule pattern expressed in A7 appears to be 4-9-6, what means: 4 semi-polar $(\mathrm{G}, \mathrm{P}, \mathrm{H}, \mathrm{W}), 9$ polar $(\mathrm{K}, \mathrm{R}, \mathrm{T}, \mathrm{S}, \mathrm{D}, \mathrm{E}, \mathrm{Q}, \mathrm{N}, \mathrm{Y})$ and 6 nonpolar (A,V,IL,C,M,F)." 18

A possible hint of the arithmetic choice of $21^{\text {st }}$ and $22^{\text {nd }}$ AAs, as well as the sources of distinction to sulfur and non-sulfur AAs in the standard GC, is found in the arrangementsystems presented in Tables 10-14. Thus, in Table 11, in the fifth position occupied by the pattern of distinction of stable multi-isotopic elements (4-9 / 11-12), in the final column we have the distinction $\{16$ (18) 20\}. On the other hand, in Table 12, built on the pattern of distinction of monoisotopic elements, we have the following situation analogously: (0-1 / 8-11) versus $\{18$ (20) 22$\}$. On the patterns of distinction of stableisotopic elements, we also have distinctions of AAs in the standard GC?!

Box 5. Explanation of Tables 11 and 12.

The result in the 5th position (taken from Table 10 as the reality of the state of the number of elements from the aspect of isotopy), in both Tables, is tested from the aspect of possible correspondence with GC. The only thing one has to do is to find for each of the four numbers its place in the set of even or odd numbers, while respecting the two Mendelian principles: continuity and minimum change. Thus, in Table 11, going up a pair of 4-9 must change to a pair of 2-7, and going down to a pair of 6-11, and so on. On the other hand, pair 11-12 going up becomes pair 9-10 and going down 13-14 etc. In Table 12, the procedure is the same, with a difference of 3 units in the pair 8-11, so the movement - up and down - is also three units each.

The result of this moving is such that in Table 11, as multiplications of number 4 , at the beginning (at the top) there are 0,1 , respectively, which is expected if the natural numbers series is really the third "hidden" alphabet of natural codes (footnote 5). That the pair of numbers 16-20, obtained in the final column, in the fifth row, is really significant can be seen by the fact that the starting pair is 0-4. But more than that. Paired with the analogous result from Table 12, with a pair of numbers 18-22, we see that the "required" quartet sequence of even numbers 16-18-20-22 is obtained, which is exactly 14 steps away from the initial sequence 2-4-6-8, which is one of the two Mendeleev quantities discussed in the organization of Table of PS (cf. Table 13 and Surveys 1-4). It is important to note that in the final column of Table 11, the change occurs by multiplying the number four. However, in Table 12 this quantity movement in the final column follows a known formula, valid both for generating stable aromatic molecules, and for electron distribution in atomic orbitals: 2, 6, 10, 14, 18, 22 (MMR, 1994b, p. 123$)^{19}$

\footnotetext{
${ }^{18}$ [Footnote 18 in source paper]: As a noteworthy is the fact that the pattern 4-9-6 corresponds to the third perfect number 496.

${ }^{19}$ MMR, 1994b, p. 123: "This paper also shows that all the relations in the chemical code and the genetic code are in accordance with periodicity and cyclicity of the natural number system with the base $\mathrm{N}_{1}=2 \mathrm{n}$ $(\mathrm{n}=1, \ldots, 6)$ and $\mathrm{N}_{2}=2(2 \mathrm{n}+1)$, where $\mathrm{n}=0,1, \ldots, 5$. These mathematical number systems lead to Golden Mean, as one of the most important law in the Nature."
} 
Additional Note 1 to Box 5. We give a small comment on the two formulas in our paper, cited in footnote 19. The first formula for the first case states $n=1$, although it is common to denote the starting point as $\mathrm{n}=0$. However, it wanted to indicate that for natural codes it is important to generate a binary tree, with two branches. Only then, it makes sense to say that for the case $n=0$ it is a whole of a binary tree. For the end point, $\mathrm{n}=6$ is stated, which meant that the only 6-bit tree corresponds to the four-letter alphabet, in terms of distinction: 4-letter alphabet, 3-letter words, 2-letter word root, 1letter word as the shortest word (cf. legend to Table 7).

For chemical elements, from the aspect of reality, the values for $n$ in the formula $N=2(2 n+1)$ are $0,1,2,3$, which corresponds to one $s$ orbital with 2 electrons; three $p$ orbitals with 6 electrons, five $d$ orbitals with 10 electrons and seven $f$ orbitals with 14 electrons. In the case of aromatic molecules, it is the number of "pi" electrons, and as it is known from organic chemistry, it makes sense to go to $\mathrm{n}=$ 5 with 22 electrons, because further stable molecules are not obtained. In our present case (Table 12) the number 22 as a last case confirms the rule valid in GC: the polysemy is on the scene when signs and designations apply (cf. footnotes 13 and 14).

Additional Note 2 to Box 5. The possible correspondence of the sequence 16-18-20-22 with GC quantities is as follows: in the set of 20 protein AAs 18 does not contain sulfur, but only four elements $(\mathrm{H}, \mathrm{C}, \mathrm{N}, \mathrm{O})$; further: out of a total of $20 \mathrm{AAs}, 16$ of them are of alanine stereochemical type, while four belong to the non-alanine types; in addition to 20 protein AAs in the standard GC, in some organisms can be found Selenocysteine, and in some other Pyrrolysine, which is a total of 22 AAs.

Additional Note 3 to Box 5. Our paper cited in footnote 19 was in the form of a poster, with all the illustrations cited in the paper (MMR, 1995), prepared for an analog scientific conference in Belgrade, a year later.

Concluding remark. In conclusion, I can only repeat what I concluded in one of the previous works: „The presented facts confirm the hypothesis ... that genetic code is a specific image of a specific mirror image. On the other hand, these facts also confirm the hypothesis of V. Shcherbak (1994) that the genetic code contains an analogy with quantum physics. All this goes in support of our hypothesis (Rakočević, 2004) that the genetic code, from the aspect of completeness of the system of its constituents (amino acid and nucleotide constituents), was still prebiotic complete - 20 protein amino acids and four nucleotide molecules in RNA." And, let me add, that after the results presented in this Supplement, I think that it makes no sense to talk only about the analogies of the genetic and chemical code, but also about their unity; the unity determined by "The Spontaneous Intelligent Design", which follows from the PSE, understood as "The Chemical Code", as it is elaborated in this Supplement. ${ }^{20}$

\footnotetext{
${ }^{20}$ MMR, 2018a, Box 4, p. 43: "With insight into the results ... one is forced to propose a hypothesis (for further researches) that here, there really is a kind of intelligent design; not the original intelligent design, dealing with the question - intelligent design or evolution ..., which is rightly criticized. ... Here, there could be such an intelligent design, which we could call 'Spontaneous Intelligent Design' (SPID) that is consistent with that design which was presented by F. Castro-Chavez (2010, p. 718) and is also in accordance with Darwinism."
} 


\section{References}

Castro-Chavez, F. (2010) The rules of variation: amino acid exchange according to the rotating circular genetic code. J. Theor. Biol. 264, 711-721.

Hawking, S., Mlodinow, L. (2005) A briefer history of time, Bantam Books, US.

Kedrov, B.M. (1977) Predictions of Mendeleev in Atomism - Unknown Elements, Atomizdat, Moscow.

Konopel'chenko, B.G., Rumer, Yu. B. (1975) Klassifikaciya kodonov v geneticheskom kode. Dokl. Akad. Nauk. SSSR. 223, 471-474.

Mendeleev, D. I. (1869) On the Relationship of the Properties of the Elements to their Atomic Weights, Zeitschrift für Chemie, 12, 405-406. [Reprinted in David M. Knight, ed., Classical Scientific Papers - Chemistry, Second Series, 1970.]

Platon (1888) Timaeus of Plato, editef with Introduction and Notes by R.D. Archer-Hind, M.A., fellow of Trinity College, Cambridge. London, New York: Macmillan and CO.

Platon (1970) Timaeus. Texte préparé et traduit par Albert Rivaud: Platon, Ouevers completes, tome X, Paris, 1970.

Popov, E.M. (1989) Strukturnaya organizaciya belkov. Nauka, Moskva.

Rakočević, M. M. (1988) Geni, molekuli, jezik, Naučna knjiga, Beograd. (www.rakocevcode.rs)

Rakočević, M.M. (1991) The coherence of the chemical and genetic code, in: Proceedings of Faculty of Science (former: Faculty of Philosophy), Chemistry Section, 2, 1991, 1-29, Niš.

Rakočević, M.M. (1994a) Logic of the Genetic Code, Naučna knjiga, Beograd www.rakocevcode.rs)

Rakočević, M.M. (1994b) Does universal consciousness exist? (Emerging Logic of the Periodic System of Elements, Genetic Code and Human Consciousness), Toward A Scientific Basis for Consciousness, An Interdisciplinary Conference, The University of Arizona, Tucson, April 12 14, 1994. Program and Abstracts, p. 123, Poster Presentations, No 62.

Rakočević, M.M. (1995) The universal consciousness and the universal code, "Consciousness Scientific Challenge for the 21st Century", Symposium, May 29 - 30, 1995, ECPD, European Centre for Peace and Development of the United Nations University for Peace, Belgrade. http://www.ecpd.org.rs/pdf/2015/books/1995/1995_ecpd_consciousness.pdf

Rakočević, M.M. (1997a) Two classes of the amino acyl-tRNA synthetases in correspondence with the Codon path cube, Bull. Math. Biol. 59 (1997) 645-648.

Rakočević, M.M. (1997b) Genetic Code as a Unique System, Studentski kulturni centar Niš and Bina - Beograd, Serbia, 1997; http://www.rakocevcode.rs

Rakočević, M.M. (1998) The genetic code as a Golden mean determined system. Biosystems, 46, 283-291. 
Rakočević, M.M. (2004a) A harmonic structure of the genetic code. J. Theor. Biol. 229, 221-234.

Rakočević, M. M. (2004b) Further generalization of Golden mean in relation to Euler's "divine" equation, FME Transactions (Faculty of Mechanical Engineering, Belgrade, Serbia), 32, 95-98, 2004; arXiv:math/0611095v1[math.GM]; http://www.rakocevcode.rs

Rakočević, M.M. (2008) Genetic Code: Four-Codon and Non-Four-Codon Degeneracy. arXiv:0802.1056v2 [q-bio.BM].

Rakočević, M.M. (2011a) Genetic Code: Four Diversity Types of Protein Amino Acids. arXiv:1107.1998v2 [q-bio.OT].

Rakočević, M.M. (2011b) Genetic code as a coherent system. Neuroquantology, 9 (4), 821-841.

Rakočević, M.M. (2015a) Po(i)etics of universal poetic structures, in: Njegoševa po(i)etika, p. 128. Pitura, Beograd.

Rakočević, M.M. (2015b) Universal poetic structures - Njegosh's work in relation to the works of Homer and Dante, Serbian literary magazine, 2015 year, pp. 137-148. (Publisher: The Serbian Writers Assotiation, Francuska 7, Belgrade, Serbia.)

Rakočević, M.M. (2017a) Golden and Harmonic Mean in the Genetic code. OSF Preprint DOI $\underline{10.31219 / \mathrm{osf} . \mathrm{io} / 2 \mathrm{pfe} 7}$

Rakočević, M.M. (2017b) Analogies of Genetic and Chemical Code (an expanded version), OSF Preprints DOI 10.31219/osf.io/mxecj

Rakočević, M.M., (2017c) Enigma of Darwin Diagram. OSF Preprint DOI

$\underline{10.31219 / \mathrm{osf} . \mathrm{io} / \mathrm{qzg} 69}$

Rakočević, M.M. (2018a) The Cipher of the Genetic Code. BioSystems 171 (2018) 31-47

Rakočević, M. M. (2018b) Analogies of Genetic and Chemical Code. Polyhedron, 153, 292-298.

Rakočević, M. M. (2019a) Protein amino acids as a complete (periodic) system. Chemia

Naissensis, Vol 2, Issue 1, pp. 1-43.

Rakočević, M. M., (2019b) Genetic code as an image of the mirror image. Part I, OSF Preprint DOI 10.31219/osf.io/xht4c

Rakočević, M. M. (2020) Genetic code as an image of the mirror image. Part II, OSF Preprint DOI $10.31219 /$ osf.io/3svpz

Rakočević, M.M., Jokić, A. (1996) Four stereochemical types of protein amino acids: synchronic determination with chemical characteristics, atom and nucleon number. J.

Theor. Biol. 183, 345-349.

Shcherbak, V. I. (1993) Twenty Canonical Amino Acids of the Genetic Code: the Arithmetical Regularities. Part I. J. Theor. Biol. 162, 399-401.

Shcherbak, V. I. (1994) Sixty-four Triplets and 20 Canonical Amino Acids of the Genetic Code: the Arithmetical Regularities. Part II. J. Theor. Biol. 166, 475-477.

Spinadel, V. W. de. (1998) From the Golden Mean to Chaos, Buenos Aires. 
Spinadel, V.W. de. (1999) The family of metallic means, Visual Mathematics,1, No. 3. (http//members.tripod.com/vismath1/spinadel/).

Sukhodolets, V.V. (1985) A sense of the genetic code: reconstruction of the prebiological evolution stage, Genetika, XXI, 10, 1589-1599 (in Russian).

Swanson, R. (1984) A unifying concept for the amino acid code. Bull. Math. Biol. 46, 187-207.

Verkhovod, A.B. (1994). Alphanumerical Divisions of the Universal Genetic Code: New Divisions Reveal New Balances. J. Theor. Biol. 170, 327-330

Woese, C.R., et al. (1966) On the fundamental nature and evolution of the genetic code. Cold Spring Harb. Symp. Quant. Biol. 31, 723-736.

Woody, Andrea, I. (2014) Chemistry's Periodic Law: Rethinking Representation and Explanation after the Turn to Practice, Routledge volume Science After the Practice Turn in Philosophy, History, and the Social Studies of Science, edited by L. Soler, S. Zwart, V.Israel---Jost, and M. Lynch.https://www.amazon.com/Science-Practice-Philosophy-History-Routledge/dp/0415722950 (Amazon Best Sellers Rank: \#10,193,928)

Academia.edu:

https://www.academia.edu/3849078/Chemistry_s_Periodic_Law_Rethinking_Representation_and Explanation_after_the_Turn_to_Practice 
Table 1. Periodic system of chemical elements with 6 groups

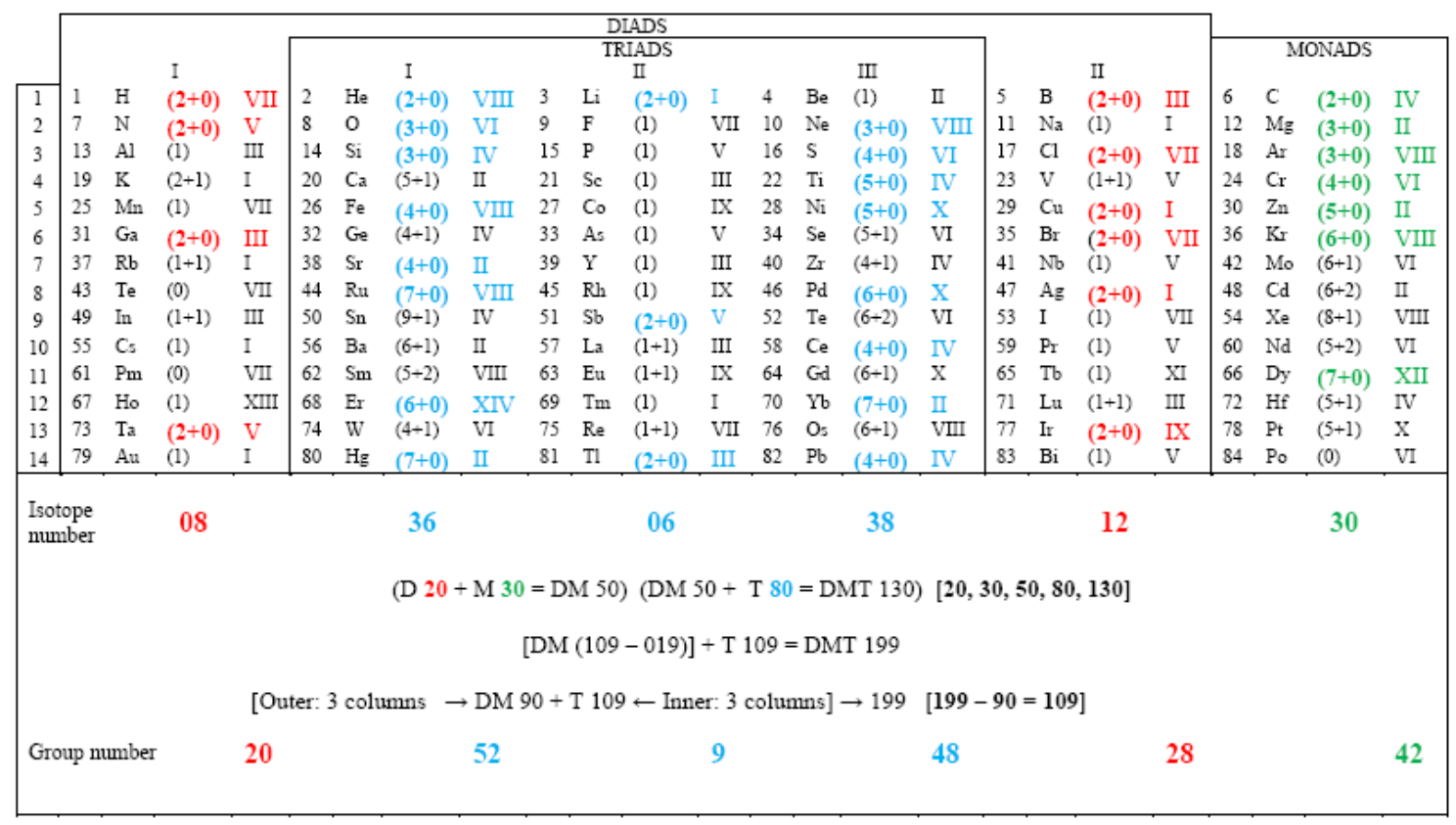

Legend to Table 1: This Table is the same one we gave in the original paper (MMR, 2018b, Table 2, p. 295). The only difference is that here the elements are given up to the limit of stability/ instability (up to Polonium); and the isotopes are given distinctly: stable plus primordially unstable. In the lower part of Table (area without elements: AWE) there is an overview of the results of summation of the number of stable isotopes in stable elements - in monads, diads and triads (upper two rows in AWE). The sums of the number of groups in which these elements are located (middle row and bottom two rows in AWE ) are also given. The result for the number of isotopes is such that it represents a correspondence with a sequence of Fibonacci numbers. The result for the sum of the number of groups ("Group number") corresponds to the result in one specific arithmetic system (Table 2). 
Table 2. An arithmetical arrangement, specific and significant for natural coding

\begin{tabular}{|c|c|c|c|c|c|c|c|}
\hline$\downarrow$ & $\downarrow$ & & $\downarrow$ & & $\uparrow$ & $\downarrow$ & $\uparrow$ \\
\hline 10 & 19 & $\rightarrow$ & 29 & + & 90 & $\rightarrow 119$ & $199-90=105$ \\
\hline 20 & 29 & $\rightarrow$ & 49 & + & 80 & $\rightarrow 129$ & $189-80=109$ \\
\hline 30 & 39 & $\rightarrow$ & 69 & + & 70 & $\rightarrow 139$ & $179-70=109$ \\
\hline 40 & 49 & $\rightarrow$ & 89 & + & 60 & $\rightarrow 149$ & $169-60=109$ \\
\hline 50 & 59 & $\rightarrow$ & 109 & + & 50 & $\rightarrow 159$ & $159-50=10$ \\
\hline 60 & 69 & $\rightarrow$ & 129 & + & 40 & $\rightarrow 169$ & $149-40=109$ \\
\hline 70 & 79 & $\rightarrow$ & 149 & + & 30 & $\rightarrow 179$ & $139-30=109$ \\
\hline 80 & 89 & $\rightarrow$ & 169 & + & 20 & $\rightarrow 189$ & $129-20=109$ \\
\hline 90 & 99 & $\rightarrow$ & 189 & + & 10 & $\rightarrow \underline{199}$ & $119-10=109$ \\
\hline
\end{tabular}

Legend to Table 2: The first column shows the first nine decades of the decimal number system, while the second column contains the first nine modular decades (by module 9). In the third column is their sum. Then, the first column is repeated in the opposite direction (the first column after the " $+"$ sign). The third column plus the fourth column give the fifth column. The sixth column consists of three subcolumns: the first represents the inverted fifth column, and the second subcolumn represents the fourth column. The third subcolumn represents the difference between the first and second subcolumn. The first result thus obtained in sixth column corresponds one hundred percent to the result of the number of groups in Table 1. [Note: The change of direction in the columns is analogous to the change of the direction of the column with amino acid molecules in: (MMR, 2019a, Table 2, p. 14, in relation to Figure 4, p. 22).] 
Table 3. The PSE group arrangement within LH and HL Boolean spaces

\begin{tabular}{|c|c|c|c|c|c|c|c|c|}
\hline$G$ & EN & GN & IN & G & EN & $\mathrm{GN}$ & IN & \\
\hline 1 & 3 & 03 & 6 & II & 5 & 10 & 26 & \multirow{4}{*}{$\begin{array}{l}\text { Lower / Higher } \\
\text { Rank (LH) }\end{array}$} \\
\hline V & 3 & 15 & 6 & VI & 3 & 18 & 11 & \\
\hline IX & 1 & 09 & 2 & $X$ & 2 & 20 & 11 & \\
\hline XIII & 0 & 0 & 0 & XIV & 1 & 14 & 06 & \\
\hline & (7) & 27 & 14 & & (11) & 62 & 54 & $89 / 68$ \\
\hline G & EN & GN & IN & G & EN & GN & IN & \\
\hline III & 3 & 09 & 6 & IV & 5 & 20 & 18 & \multirow{3}{*}{$\begin{array}{l}\text { Higher / Lower } \\
\text { rank (HL) }\end{array}$} \\
\hline VII & 3 & 21 & 6 & VIIII & 6 & 48 & 25 & \\
\hline XI & 0 & 0 & 0 & XII & 1 & 12 & 07 & \\
\hline & (6) & 30 & 12 & & (12) & 80 & 50 & $110 / 62$ \\
\hline
\end{tabular}

Legend to Table 3: The meaning of the labels is as follows: $\mathrm{G}$ - the group in PSE; EN - number of chemical elements in the given group; GN - "Group number" $(1 \times 3=3 ; 5$ × $3=15 ; 9 \times 1=9$ etc.); IN - number of stable isotopes ("Isotope number"). The explanation of relationships in the text. Test for the first quadrant according to the data in Survey 9 and 10: First group $\mathrm{Li}_{2}+\mathrm{Cu}_{2}+$ $\mathrm{Ag}_{2}=6$; fifth group $\mathrm{N}_{2}+\mathrm{Sb}_{2}+\mathrm{Ta}_{2}=6$; deveta grupa $\mathrm{Ir}_{2}=2$. The remaining three quadrants can be tested in the same way. 
Table 4. Fibonacci "triangles" (I)

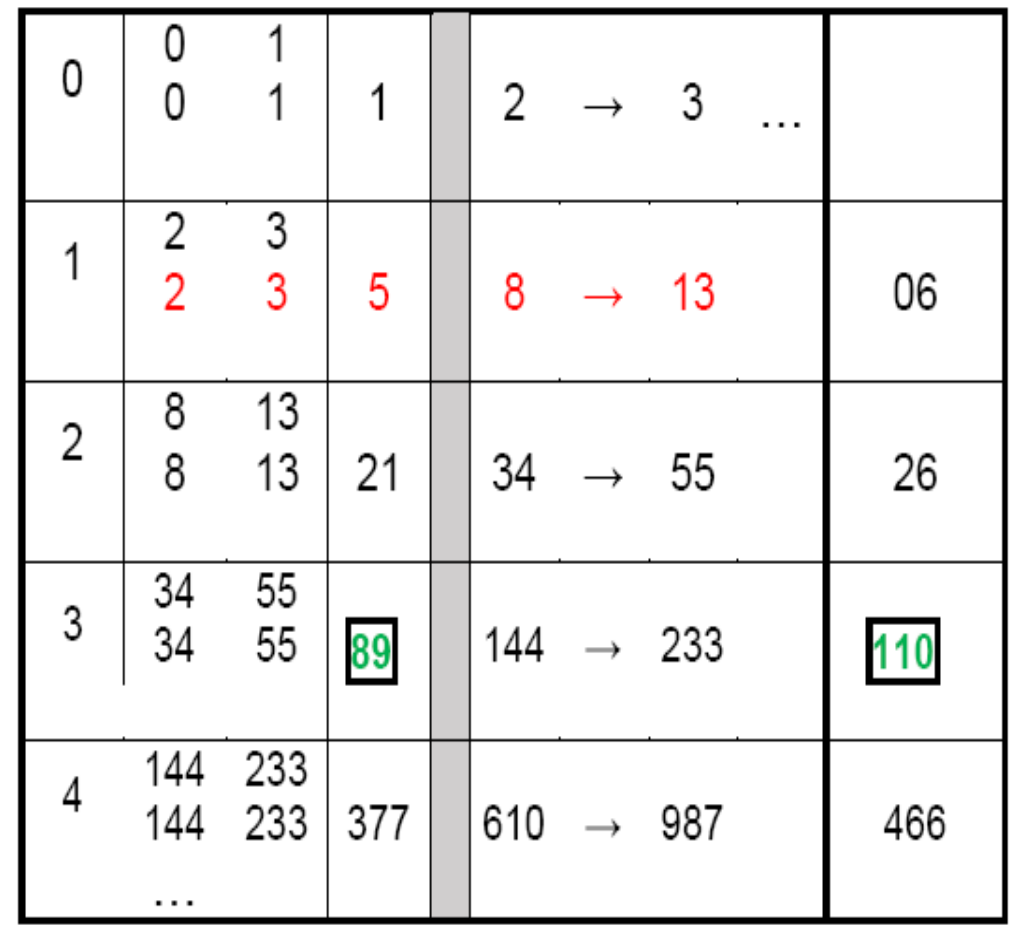

Explanation in the text. 
Table 5. Fibonacci "triangles" (II)

\begin{tabular}{|c|c|c|c|c|c|c|c|}
\hline 0 & $\begin{array}{l}0 \\
0\end{array}$ & $\begin{array}{l}-1 \\
-1\end{array}$ & -1 & -2 & $\rightarrow-3$ & $\ldots$ & \\
\hline 0 & $\begin{array}{l}0 \\
0\end{array}$ & $\begin{array}{l}1 \\
1\end{array}$ & 1 & & $\rightarrow 3$ & 02 & \\
\hline 1 & $\begin{array}{l}2 \\
2\end{array}$ & $\begin{array}{l}3 \\
3\end{array}$ & 5 & 8 & $\rightarrow 13$ & 06 & 08 \\
\hline 2 & $\begin{array}{l}8 \\
8\end{array}$ & $\begin{array}{l}13 \\
13\end{array}$ & 21 & 34 & $\rightarrow 55$ & 26 & 32 \\
\hline 3 & $\begin{array}{l}34 \\
34\end{array}$ & $\begin{array}{l}55 \\
55\end{array}$ & 89 & 144 & $\rightarrow 233$ & 110 & 136 \\
\hline 4 & $\begin{array}{l}144 \\
144\end{array}$ & $\begin{array}{l}233 \\
233\end{array}$ & 377 & 610 & $\rightarrow 987$ & 466 & 576 \\
\hline 5 & $\begin{array}{l}610 \\
610\end{array}$ & $\begin{array}{l}987 \\
987\end{array}$ & 1597 & 2584 & $\rightarrow 4181$ & 1974 & 2440 \\
\hline
\end{tabular}

Legend to Table 5: Everything is the same as in the previous Table 4, except that the zeroth triangle from the area of negative Fibonacci triangles is added here at the beginning. 
Table 6. The "hidden" Fibonacci sequence (I)

\begin{tabular}{|c|c|c|c|c|c|}
\hline 8 & 32 & 136 & 576 & 2440 & \\
$1 \times 8$ & $4 \times 8$ & $17 \times 8$ & $72 \times 8$ & $305 \times 8$ & \\
1 & 4 & 17 & 72 & 305 & $\ldots$ \\
\hline & 3 & 13 & 55 & 233 & \\
& & & & & \\
& $3(5,8)$ & $13(21,34)$ & $55(89,144)$ & 233 & \\
\hline
\end{tabular}

Legend to Table 6: The "hiding" of some members of the Fibonacci sequence over the multiples of number 8 corresponds to the "hiding" of number 50 for the number of isotopes in monadsdiads-triads in Table 1: it does not appear as the quantity of any of the three possible entities but as the sum of monads and diads. (Cf. the generation of the first and the second Fibonacci triangle within Table 4, highlighted in red color.) 
Table 7. The "hidden" Fibonacci sequence (II)

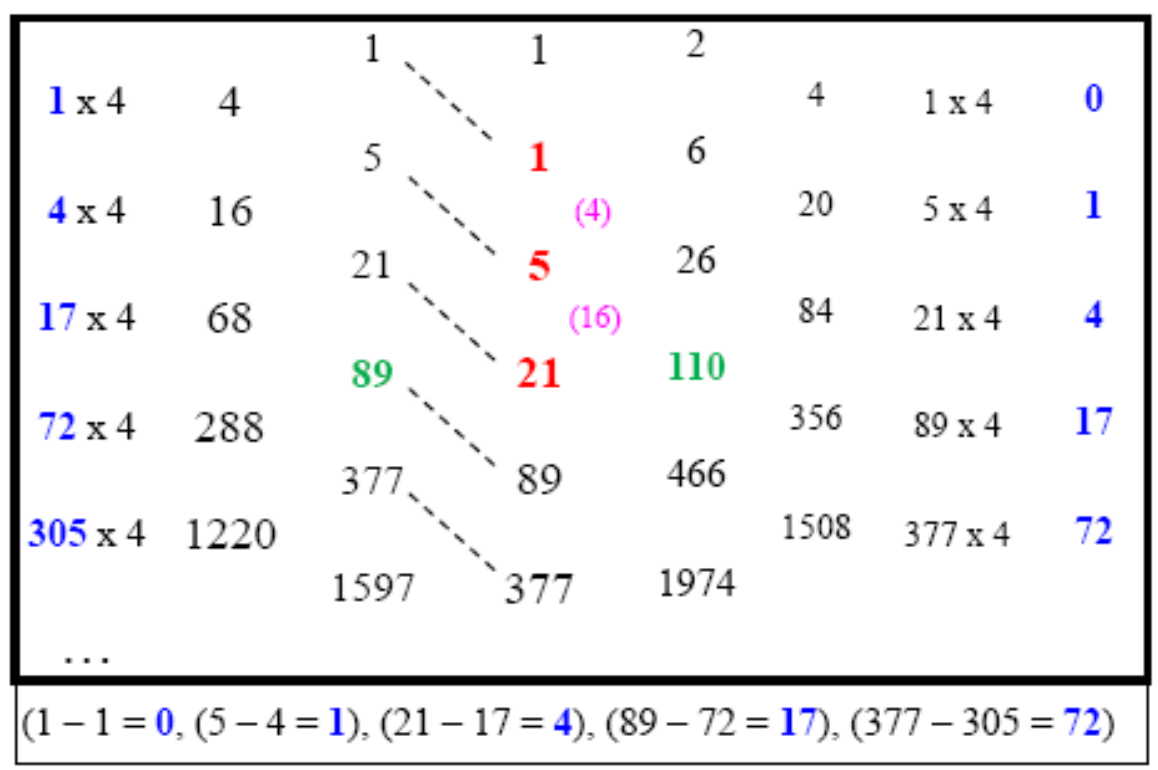

Legend to Table 7: Further more detailed of the relationships shown in the previous Table 6. The numbers marked in red color correspond to one third of the corresponding binary tree. In case of number 1 we have a 2-bit binary tree (0-3), with 4 one-letter words. In the case of number 5, it is 4-bit binary tree (0-15) with 16 two-letter words. In the case of number 21, there is a 6-bit binary tree with 64 three-letter words on the scene. That's how we find it in GC. Going further none of this logic is possible because 89 is not a third of any binary tree. 
Table 8. The "hidden" Fibonacci sequence (III)

\begin{tabular}{|c|c|c|c|c|c|}
\hline 1 & 1 & 2 & $\rightarrow$ & 4 & $\begin{array}{c}\mathbf{1} \\
(1,2)\end{array}$ \\
\hline 5 & 1 & 6 & $\rightarrow$ & 12 & $\begin{array}{c}3 \\
(5,8)\end{array}$ \\
\hline 21 & 5 & 26 & $\rightarrow$ & 52 & $\begin{array}{c}13 \\
(21,34)\end{array}$ \\
\hline 89 & 21 & 110 & $\rightarrow$ & 220 & $\begin{array}{c}55 \\
(89,144)\end{array}$ \\
\hline 377 & 89 & 466 & $\rightarrow$ & 932 & $\begin{array}{c}233 \\
(377,610)\end{array}$ \\
\hline $\begin{array}{c}1597 \\
\ldots\end{array}$ & 377 & 1974 & $\rightarrow$ & 3948 & 987 \\
\hline
\end{tabular}

Legend to Table 8: Further detailing the relationships shown in the previous Table 7. (Notice that the number 220 is the first friendly number.) 
Table 9. The entity "independence" of atoms and nucleons in GCT

\begin{tabular}{|c|c|c|c|c|c|}
\hline $\begin{array}{l}F_{91} \\
L_{57} \\
\end{array}$ & $S_{31}$ & $Y_{107}$ & $\begin{array}{c}\mathrm{C}_{47} \\
\mathrm{~W}_{130}\end{array}$ & & $\begin{array}{c}1443 \\
\times 6\end{array}$ \\
\hline $\mathrm{L}_{57}$ & $P_{41}$ & $\begin{array}{l}\mathrm{H}_{81} \\
\mathrm{Q}_{72}\end{array}$ & $R_{100}$ & & 8658 \\
\hline $\begin{array}{c}I_{57} \\
M_{75}\end{array}$ & $\mathrm{~T}_{45}$ & $\begin{array}{l}\mathrm{N}_{58} \\
\mathrm{~K}_{72} \\
\end{array}$ & $\begin{array}{l}\mathrm{S}_{31} \\
\mathrm{R}_{100}\end{array}$ & & $\begin{array}{c}\downarrow \\
7770 \\
0888\end{array}$ \\
\hline$V_{43}$ & $A_{15}$ & $\begin{array}{l}D_{59} \\
E_{73}\end{array}$ & $\mathrm{G}_{01}$ & & 8658 \\
\hline 380 & 132 & 522 & 409 & $\rightarrow$ & 1443 \\
\hline $\begin{array}{l}2 \mathrm{~F}_{14} \\
2 \mathrm{~L}_{13} \\
\end{array}$ & $4 S_{05}$ & $2 Y_{15}$ & $\begin{array}{l}2 \mathrm{C}_{05} \\
1 \mathrm{~W}_{18}\end{array}$ & & $\begin{array}{l}0006 \\
0028\end{array}$ \\
\hline $4 L_{13}$ & $4 \mathrm{P}_{08}$ & $\begin{array}{l}2 \mathrm{H}_{11} \\
2 \mathrm{Q}_{11}\end{array}$ & $4 \mathrm{R}_{17}$ & & $\begin{array}{r}0496 \\
8658 \\
\end{array}$ \\
\hline $\begin{array}{l}3 \mathrm{I}_{13} \\
1 \mathrm{M}_{11}\end{array}$ & $4 T_{08}$ & $\begin{array}{l}2 \mathrm{~N}_{08} \\
2 \mathrm{~K}_{15}\end{array}$ & $\begin{array}{l}2 S_{05} \\
2 R_{17}\end{array}$ & & $\begin{array}{l}0111(7) \\
1000(8)\end{array}$ \\
\hline $4 V_{10}$ & $4 \mathrm{~A}_{04}$ & $\begin{array}{l}2 \mathrm{D}_{07} \\
2 \mathrm{E}_{10}\end{array}$ & $4 G_{01}$ & & \\
\hline 196 & 100 & 154 & 144 & $\rightarrow$ & 594 \\
\hline \multicolumn{3}{|c|}{$61 \times 9=549$} & \multicolumn{3}{|c|}{$549+594=1143$} \\
\hline \multicolumn{6}{|c|}{$1443-1143=300$} \\
\hline
\end{tabular}

Legend to Table 9: Genetic Code Table with 23 amino acid molecules (above) and 61 amino acid molecules (below). In the first case, the index indicates the number of nucleons in the side chain of amino acid, and in the second case, the number of atoms. To the right are the calculations, with the indicated relations with the sum of the first four perfect numbers and the Boolean hypercube. The difference between the two systems ("chalk" and "cheese") is 300, which is the vertices "3" (in binary number system: 11) of the logical square given in Table A10. 
Table 10. Number of stable isotopes: symmetries and balances

\begin{tabular}{|c|c|c|c|c|c|c|c|}
\hline & \multicolumn{3}{|c|}{ Poly } & \multicolumn{3}{|c|}{ Mono } & \\
\hline & 0 & $E$ & & 0 & $E$ & & \\
\hline$(s, p)$ & 9 & 11 & $(5 \times 4)$ & 8 & 1 & 9 & (29) \\
\hline \multirow[t]{2}{*}{$(\mathrm{d}, \mathrm{f})$} & 4 & 12 & $(4 \times 4)$ & 11 & 0 & 11 & (27) \\
\hline & 13 & 23 & 36 & 19 & 01 & 20 & 56 \\
\hline$[(1)$ & 19 & $\mathrm{x} 4=$ & & {$[(23+$} & $1=8$ & $3=$ & \\
\hline
\end{tabular}

Legend to Table 10: Labels as follows; Poly - Poly-isotopic elements; Mono - Monoisotopic elements; O - Odd elements; E - Even elements. On the left side of the Table it is shown that the number of stable multi-isotopic elements is 36 , exactly as we presented in the previous paper (MMR, 2018b, Survey 3a, p. 296). On the right side, there is a well-known fact that there are 20 monoisotopic elements. There are 56 total stable elements, namely: $28+1$ elements of $s, p$ type (non-transition elements) and 28-1 elements of $d$, $f$ type (transition plus inner transition elements). [Cf. Survey B5: on the total number of stable isotopes (56).] 
Table 11. The arithmetical source of distinctions of stable poly-isotopic elements

\begin{tabular}{|c|c|c|c|c|}
\hline$(1)$ & 1 & 3 & $(4 \times 1)$ & {$[4]$} \\
& -4 & 4 & $(4 \times 0)$ & {$[0]$} \\
\hline$(2)$ & 3 & 5 & $(4 \times 2)$ & {$[8]$} \\
& -2 & 6 & $(4 \times 1)$ & {$[4]$} \\
\hline$(3)$ & 5 & 7 & $(4 \times 3)$ & {$[12]$} \\
& 0 & 8 & $(4 \times 2)$ & {$[8]$} \\
\hline$(4)$ & 7 & 9 & $(4 \times 4)$ & {$[16]$} \\
& 2 & 10 & $(4 \times 3)$ & {$[12]$} \\
\hline$(5)$ & 9 & 11 & $(4 \times 5)$ & {$[\mathbf{2 0}]$} \\
& 4 & 12 & $(4 \times 4)$ & {$[\mathbf{1 6}]$} \\
\hline$(6)$ & 11 & 13 & $(4 \times 6)$ & {$[24]$} \\
$\ldots$ & 6 & 14 & $(4 \times 5)$ & {$[20]$} \\
\hline
\end{tabular}

Legend to Table 11: The "Aufbau" of the arithmetic arrangement-system in relation to the pattern of distinction (in the fifth position) of poly-isotopic stable elements, given in Table 10. One can notice that the multiples of number 4 in the first to last column correspond to the values of the variables in the generalized golden mean equation (MMR, 2004b, Table 1, p. 96). (See Explanation of Tables 11 and 12 in Box 5.) 
Table 12. The arithmetical source of distinctions of monoisotopic elements

\begin{tabular}{|c|c|c|c|c|}
\hline$(1)$ & -8 & 9 & 1 & {$[02]$} \\
& -5 & 8 & 3 & {$[06]$} \\
\hline$(2)$ & -4 & 7 & 3 & {$[06]$} \\
& -1 & 6 & 5 & {$[10]$} \\
\hline$(3)$ & 0 & 5 & 5 & {$[10]$} \\
& 3 & 4 & 7 & {$[14]$} \\
\hline$(4)$ & 4 & 3 & 7 & {$[14]$} \\
& 7 & 2 & 9 & {$[18]$} \\
\hline$(5)$ & 8 & 1 & 9 & {$[18]$} \\
& 11 & 0 & 11 & {$[22]$} \\
\hline$(6)$ & 12 & -1 & 11 & {$[22]$} \\
& 15 & -2 & 13 & {$[26]$} \\
\hline (7) & 16 & -3 & 13 & {$[26]$} \\
& 19 & -4 & 15 & {$[30]$} \\
\hline
\end{tabular}

Legend to Table 12: The "Aufbau" of the arithmetic arrangement-system in relation to the pattern of distinction of monoisotope stable elements, given in Table 10. 
Table 13. Genetic and chemical code unity sequences (I)

\begin{tabular}{|cccc|c|c|}
\hline 2 & 4 & 6 & 8 & $(20)$ & \\
14 & 14 & 14 & 14 & & \\
16 & 18 & 20 & 22 & $\rightarrow$ & 76 \\
$32 / 64$ & $32 / 68$ & $32 / 72$ & $32 / 76$ & & $\downarrow$ \\
48 & 50 & 52 & 54 & $(204)$ & 19 \\
14 & 14 & 14 & 14 & & $\times$ \\
62 & 64 & 66 & 68 & $(260)$ & 4 \\
\hline $4 \times 32=128$ & $64+68+72+76=280$ \\
\hline \multicolumn{7}{|c|}{$128+280=2 \times \underline{204}$} \\
\hline
\end{tabular}

Legend to Table 13: Significant quartet sequences in relation to the genetic and/or chemical code: 2-4-6-8 as number of amino acid molecules within four diversity types of AAs (MMR, 2011b, Fig 2); 48-50-52-54 from Table A2 in this paper; 62-64-66-68 from Table 3 (last row) in this paper. The "between" sequence 16-18-20-22 from the fifth position of the arrangements given in Tables 11 and 12. The key quantities are highlighted in green: 20 amino acid molecules; 204 atoms in 20 amino acid molecules (their side chains); 260 as a double value of the number of stable isotopes in 36 stable poly-isotopic elements. The quantity "19" as the "modularity threshold" of the decades series in the decimal number system (Table 2, second column). 
Table 14. Genetic and chemical code unity sequences (II)

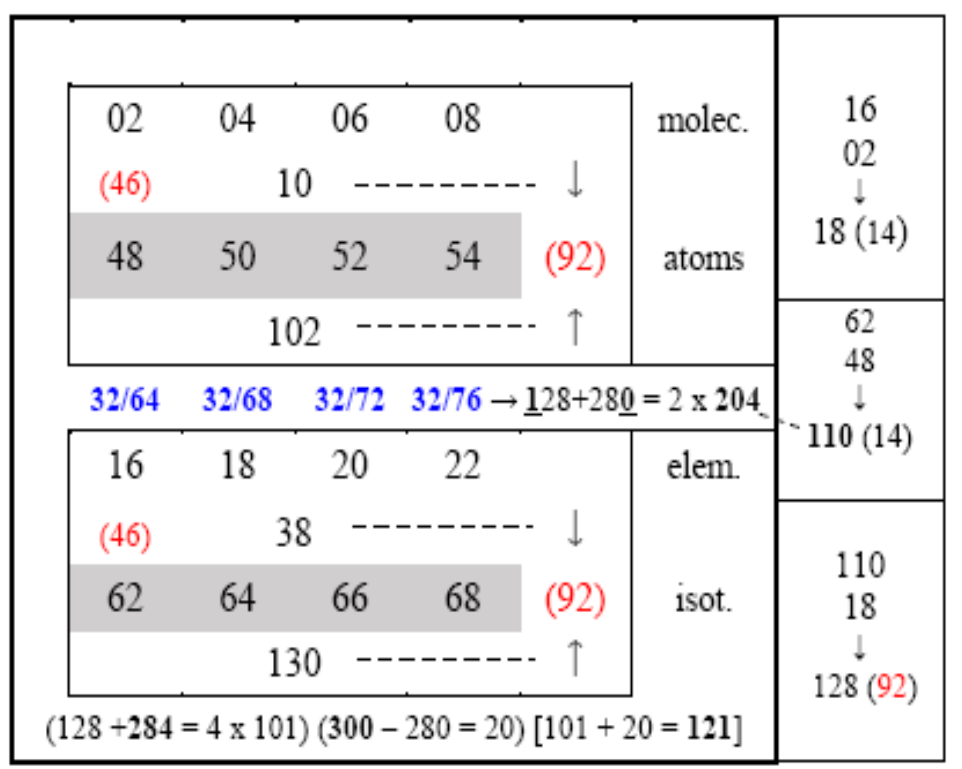

Legend to Table 14: Everything is the same as in the previous Table 13, except that here the sequences are distinguished by natural law: the upper two refer to the Genetic Code (GC), and the lower two to the chemical code (CC). Significant quantities from the key logical square of Nature (204-220-284-300) contained in the system arrangement in Table A8, are also "hidden" here: 204 doubled, 220 halved; 284 contained in relation to quantities 128 and 101; and 300 contained in the relation of quantities 280 and 20. At the end, there is the number 121, the square of the number 11 and the tenth part of the fourth friendly number - the second member of the second friendly pair (1184 - 1210). [The direct correspondence of perfect and friendly numbers is realized precisely through the third friendly number, as shown in Tables B7, B8 and B9. We recall that the two key results of V. Shcherbak (1994) for the number of nucleons in 23 AAs molecules also contain the correspondence of perfect and friendly numbers: the total number of nucleons in 23 amino acid side chains is 1443, which is one sixth of the sum of the first four perfect numbers; and the number of nucleons in the 8 "heads" of four-codon AAs is 592, which is half of the third friendly number.] 
Survey 1. Relations among specific four-member sequences

of even natural numbers (I)

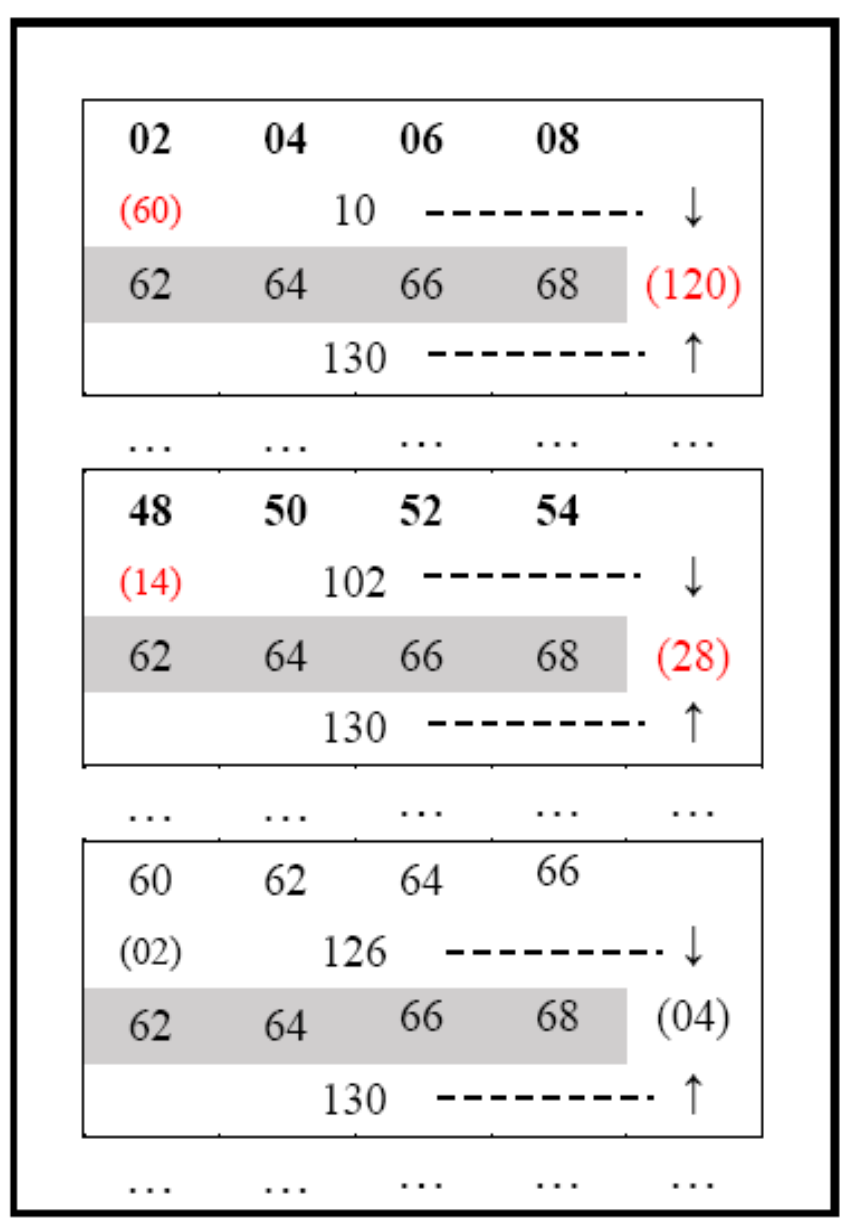

Legend to Survey 1: The sequence 62-64-66-68 corresponds to the number of stable isotopes in Table 3; the sequence 48-50-52-54 corresponds to the number of atoms in the amino acid molecules in GC, as shown herein in Table A2 with green color; finally, the sequence 2-4-6-8 corresponds to the number of AAs in the four amino acid types of diversity, as we presented in: MMR, 2011b, Table 8, p. 830. The correspondence with the first and second perfect numbers (6 $\& 28$ ) is immediately obvious. [Note 1: The difference between the quartets is indicated only at the first member in both sequences (for example: $62-02=60$, etc.); the difference between the sum of each of the two central members is given at the end, between the dashed lines and the two arrows (for example: $130-10=120$, etc. Note 2: On the perfect and friendly numbers as determinants of GC see in: MMR, 2019a, Survey B2 and Footnote 15.] 
Survey 2. Relations among specific four-member sequences of even natural numbers (II)

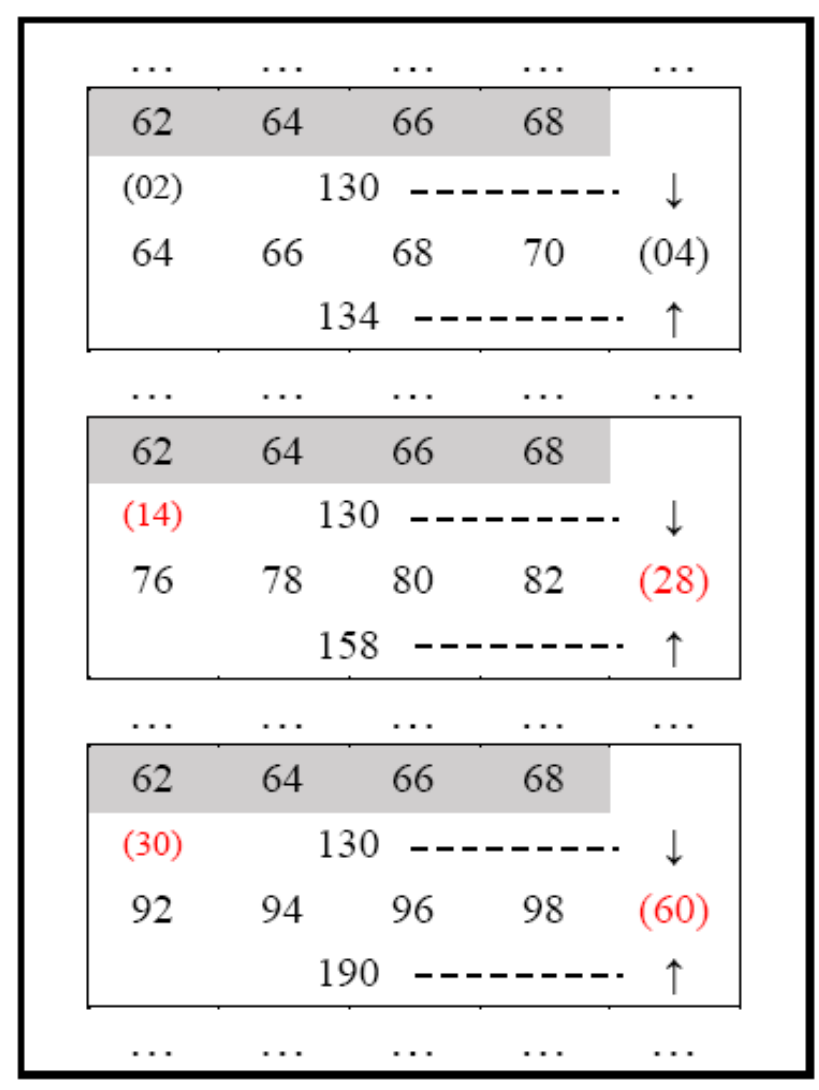

Legend to Survey 2: Everything is the same as in Survey 1, except that here the direction of the sequences is downwards, starting from the sequence 62-64-66-68 to the last in the set of two-digit natural numbers. As can be seen, the correspondence with the first and second perfect number is immediately obvious here as well. 
Survey 3. Relations among specific four-member sequences of even natural numbers (III)

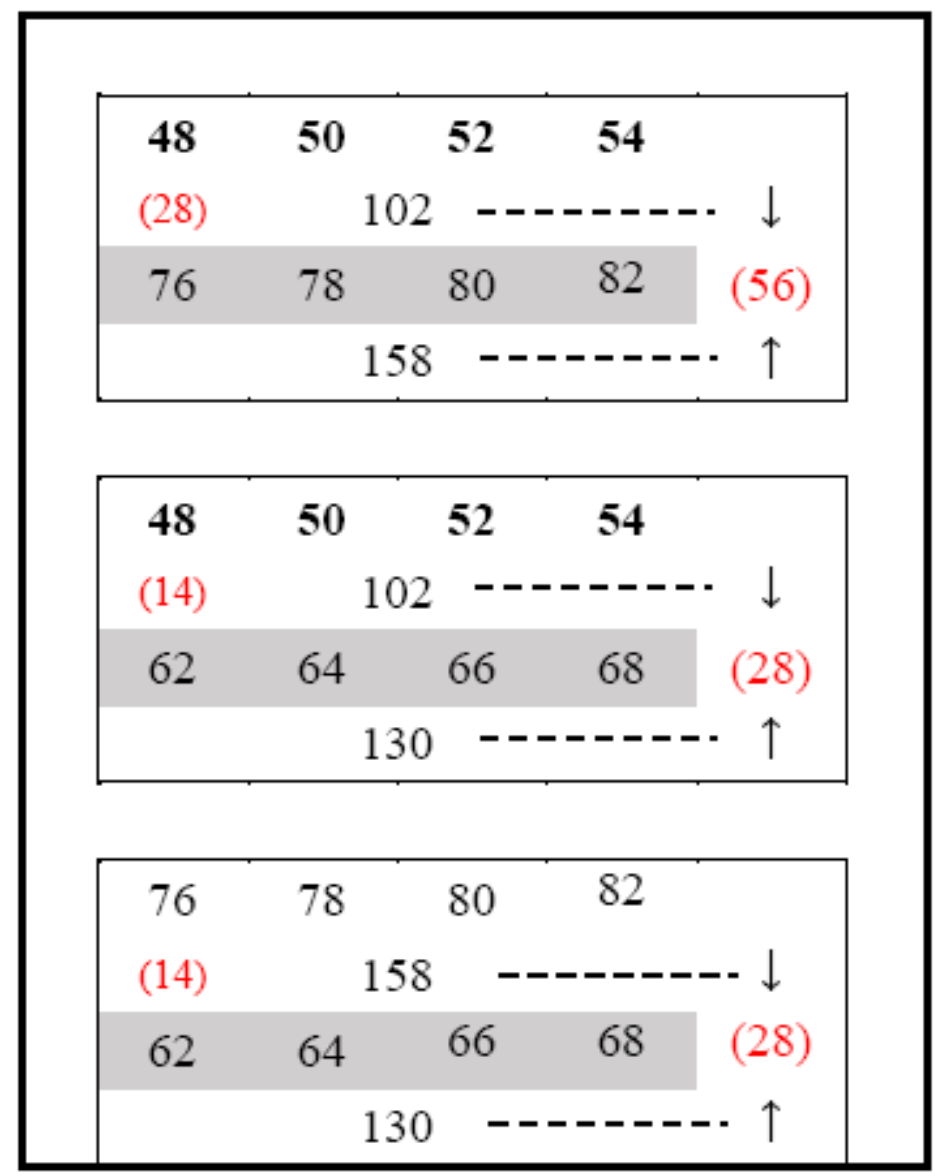

Legend to Survey 3: Examining the relationship of the sequence 48-50-52.54 in the genetic code and the sequence 62-64-66-68 in the chemical code, as well as in Survey 1. The only difference is that there is the direction of growth of the sequences upwards, and here downwards. As can be seen, the correspondence with the first and second perfect number is immediately obvious here as well. [About perfect and friendly numbers see in: MMR, 2018a: Footnote 10, p. 38; 2019a: Footnote 15, p. 38 , then pp. 22, 23, 34, 35.] 
Survey 4. Quantity relations in chemical and genetic code

\begin{tabular}{|c|c|c|c|c|c|c|c|c|c|}
\hline 42 & 44 & 46 & 48 & 02 & 04 & 06 & 08 & \multirow{3}{*}{$\rightarrow$} & \multirow{4}{*}{$\begin{array}{c}60+40=100 \\
62-42=20 \\
14 \& 06\end{array}$} \\
\hline 60 & 60 & 60 & 60 & 40 & 40 & 40 & 40 & & \\
\hline 62 & 64 & 66 & 68 & 42 & 44 & 46 & 48 & & \\
\hline 10 & 10 & 10 & 10 & 06 & 06 & 06 & 06 & $\rightarrow$ & \\
\hline 72 & 74 & 76 & 78 & 48 & 50 & 52 & 54 & & \multirow{2}{*}{$\begin{array}{l}60=50+10 \\
40=50-10\end{array}$} \\
\hline \multicolumn{4}{|c|}{$64+66=130$ (poly) } & \multicolumn{4}{|c|}{$50+52=102$} & & \\
\hline \multicolumn{4}{|c|}{$\begin{array}{l}74+76=150(\text { poly }+ \text { mono }) \\
72+78=150(300)\end{array}$} & \multicolumn{4}{|c|}{$\begin{array}{l}42+44+46+48=180 \\
48+50+52+54=204\end{array}$} & & $\begin{array}{l}180 \\
204 \\
384\end{array}$ \\
\hline \multicolumn{5}{|c|}{$\begin{array}{l}\mathrm{IN} \rightarrow[\mathrm{D}(10+1)]+[\mathrm{T}(10-1)]=20(\text { mono }) \\
\mathrm{GN} \rightarrow \mathrm{D} 59+\mathrm{T} 42=101[101+199=300] \\
\text { (monoisotopic elements) } \quad \text { (mono + poly) }\end{array}$} & \multicolumn{5}{|c|}{$\begin{array}{l}\mathrm{IN} \rightarrow[\mathrm{s}, \mathrm{p}(65-10)]+[\mathrm{d}, \mathrm{f} \rightarrow 65+10]=130 \\
\mathrm{GN} \rightarrow[\mathrm{s}, \mathrm{p}(90+11)]+[\mathrm{d}, \mathrm{f} \rightarrow 109-11]=130 \\
(\text { polyisotopic }) \text { GN } \rightarrow(101+101)+(109-11)=300\end{array}$} \\
\hline
\end{tabular}

Legend to Survey 4: A detailed explanation is given in the text. However, I would like to draw the attention to the quantity "384", which will be in a (possible) relation with the same quantity in Table B4; also to the quantity "20" which appears as the key quantity in the determination of one specific arithmetical arrangement in Table B5. Finally, the appearance of the quantity "100" in the presented way, conditioned the search for the arithmetical arrangement in the next Survey 5, which includes the quantity "130", the number of stable isotopes in 36 stable chemical elements. (See Explanation in Box 2.) 
Survey 5. Relations within a set of specific three-digit natural numbers

\begin{tabular}{|l|l|}
\hline $130+102=(1 \times 144)+88$ \\
$130+158=(2 \times 144)+00$ \\
$102+158=(2 \times 130)$ \\
$130=144-14(144=12 \times 12)$ \\
\hline \multicolumn{2}{|c|}{} \\
$100-10=090$ \\
$111-11=100$ \\
$122-12=110$ \\
$133-13=120$ \\
$144-14=130\left(5^{\text {th }} / 6^{\text {th }}\right)$ & $65 \times 2$ \\
$155-15=140$ & $55 \times 2$ \\
$166-16=150$ & $50 \times 2$ \\
$177-17=160$ & $55 \times 2$ \\
$188-18=170$ & $70 \times 2$ \\
$199-19=180$ & $75 \times 2$ \\
& $80 \times 2$ \\
& $85 \times 2$ \\
& $90 \times 2$ \\
&
\end{tabular}

Legend to Survey 5: The upper part shows the relations between the quantities found in Survey 3. Number 102 as half of 204 atoms in the 20 canonical AAs, in their side chains. Number 130 as the number of stable isotopes in 36 stable elements. The number 158 is the corresponding quantity in sequence 76-78-80-82, corresponding to both coding sequences (48-50-52-54 in GC and 62-64-66-68 in CC), as shown in Survey 3. The lower part shows all three-digit numbers, which in the first position (going from left to right) have the number "1", and in the second and third positions the numbers from "00" to "99". In the specific relation with numbers $10-19$, the number 130 (which represents the number of stable isotopes) is the only one that is in the relation of similarity and self-similarity with numbers 5 and 6 , with their order 5 th and 6th from top to bottom and from bottom to top, respectively. The uniqueness of the numbers 5 and 6 is that they are only two adjacent numbers whose squares differ by 11 within the Periodic System of Numbers, in the decimal number system (MMR, 2019a, Figure A1; here: Table A4). Half of the number 130 (65) also corresponds to the said similarity and self-similarity. 
Survey 6. Specific Arithmetical Natural-Code Sequences, SANCS (I)

\begin{tabular}{|c|c|c|c|c|c|c|c|c|}
\hline 10 & 12 & 14 & 16 & $\ldots$ & 42 & 44 & 46 & 48 \\
\hline 14 & 14 & 14 & 14 & & 14 & 14 & 14 & 14 \\
\hline 24 & 26 & 28 & 30 & $\ldots$ & 56 & 58 & 60 & 62 \\
\hline 34 & 38 & 42 & 46 & $\ldots$ & 98 & 102 & 106 & 110 \\
\hline & & & $\begin{array}{c}\downarrow \\
50\end{array}$ & (144) & $\begin{array}{c}\uparrow \\
94\end{array}$ & & & \\
\hline & & & 54 & $\begin{array}{l}(144) \\
(\end{array}$ & 90 & & & \\
\hline & & & 58 & (144) & 86 & & & \\
\hline & & & 62 & $\begin{array}{l}(144) \\
\end{array}$ & 82 & & & \\
\hline & & & $\begin{array}{l}60 \\
70 \\
70\end{array}$ & $\stackrel{(144)}{\rightarrow}$ & $\begin{array}{l}78 \\
74\end{array}$ & & & \\
\hline & & & & & & & & \\
\hline
\end{tabular}

Legend to Survey 6: The path from the first two-digit number, and its counterpart, created by increasing by Mendeleev's quantity "14", to the first members of the quartet sequence GC and quartet sequence CC, removed from the first and last row in Survey 3. Quantity "144" appears whose appearance corresponds to his appearance in the previous Survey 5 (red color).

Survey 7. Specific Arithmetical Natural-Code Sequences, SANCS (II)

\begin{tabular}{|cccccccccccccccc|}
\hline 48 & 50 & 52 & 54 & 56 & 58 & 60 & 62 & 64 & 66 & 68 & 70 & 72 & $\ldots$ \\
14 & 14 & 14 & 14 & 14 & 14 & 14 & 14 & 14 & 14 & 14 & 14 & 14 & $\ldots$ \\
62 & 64 & 66 & 68 & 70 & 72 & 74 & 76 & 78 & 80 & 82 & 84 & 86 & $\ldots$ \\
110 & 114 & 118 & 122 & 126 & 130 & 134 & 138 & 142 & 146 & 150 & 154 & 158 & $\ldots$ \\
\hline \multicolumn{1}{c}{$(110+150=130 \times 2),(114+146=130 \times 2),(118+142=130 \times 2),(122+138=130 \times 2)$} \\
\\
{$[48+76=124][(48+82=130),(50+80=130),(52+78=130),(54+76=130)]$}
\end{tabular}

Legend to Survey 7: The continuation of the path started in previous Survey 6, reveals a symmetrical position of quantity 130 . Here, for the chemical code the middle member is selected, but in GC the middle member is not selected (Table A3 in relation to Table A4. [Tables A5 and A6 show how Table A3 is formed, in connection to Table 4.] 
Survey 8. Relations between square of a given number and its sums with the follower

\begin{tabular}{|c|c|c|c|c|c|}
\hline (1) & $\begin{array}{l}1 \times 1= \\
1+2=\end{array}$ & $\begin{array}{l}1 \\
3\end{array}$ & $(2 \times 2)$ & (10) & \\
\hline (2) & $\begin{array}{l}2 \times 2= \\
2+3=\end{array}$ & $\begin{array}{l}4 \\
5\end{array}$ & $(3 \times 3)$ & (9) & \\
\hline (3) & $\begin{array}{l}3 \times 3= \\
3+4=\end{array}$ & $\begin{array}{l}9 \\
7\end{array}$ & $(4 \times 4)$ & (8) & \\
\hline (4) & $\begin{array}{l}4 \times 4= \\
4+5=\end{array}$ & $\begin{array}{c}16 \\
9\end{array}$ & $(5 \times 5)$ & (7) & \\
\hline (5) & $\begin{array}{l}5 \times 5= \\
5+6=\end{array}$ & $\begin{array}{l}25 \\
11\end{array}$ & $(6 \times 6)$ & (6) & $5^{\text {th }} / 6^{\text {th }}$ \\
\hline (6) & $\begin{array}{l}6 \times 6= \\
6+7=\end{array}$ & $\begin{array}{l}36 \\
13 \\
\end{array}$ & $(7 \times 7)$ & (5) & $6^{\text {th }} / 5^{\text {th }}$ \\
\hline (7) & $\begin{array}{l}7 \times 7= \\
7+8=\end{array}$ & $\begin{array}{l}49 \\
15\end{array}$ & $(8 \times 8)$ & (4) & \\
\hline (8) & $\begin{array}{l}8 \times 8= \\
8+9=\end{array}$ & $\begin{array}{l}64 \\
17\end{array}$ & $(9 \times 9)$ & (3) & \\
\hline (9) & $\begin{array}{l}9 \times 9= \\
8+9=\end{array}$ & $\begin{array}{l}81 \\
17\end{array}$ & $(9 \times 9)$ & (2) & \\
\hline (10) & $\begin{array}{l}10 \times 10= \\
10+11=\end{array}$ & $\begin{array}{c}100 \\
21\end{array}$ & $(11 \times 11)$ & (1) & \\
\hline
\end{tabular}

Legend to Survey 8: In the sixth row we find the relations of the number 6 (the first perfect number) and its successor, the number 7, which boils down to 36:13, which corresponds to the number of stable chemical elements (36) and the number of their stable isotopes (130), as shown in Table 1. In addition, the specific relationship of the squares of numbers 5 and 6 and their position in the given arithmetic system is shown, which is the realization of the principle of selfsimilarity. [On the validity of the principle of self-similarity in GC see in: MMR, 2004, Section 7.1, p. 231.] 
Survey 9. "Element number", "Stable isotope number" and

"Groupe number" in (s, p) stable poly-isotopic elements

\begin{tabular}{|c|c|c|c|c|c|}
\hline 1 & $\mathrm{I} \mathrm{Li}_{2}$ & 2 & 1 & 1 & \multirow{4}{*}{$10 \pm 1$} \\
\hline 3 & III B $\mathrm{B}_{2}, \mathrm{III} \mathrm{Ga}, \mathrm{III} \mathrm{T1_{2 }}$ & 6 & 3 & 9 & \\
\hline 5 & $\mathrm{~V} \mathrm{~N}_{2}, \mathrm{~V} \mathrm{Sb}_{2}$ & 4 & 2 & 10 & \\
\hline \multirow[t]{2}{*}{7} & VII $\mathrm{H}_{2}$, VII $\mathrm{Cl}_{2}$, VII $\mathrm{Br}_{2}$ & 6 & 3 & 21 & \\
\hline & & 18 & 9 & 41 & $51-10$ \\
\hline 2 & "II Mar II S & 7 & 2 & 4 & \multirow{5}{*}{$\begin{array}{c}37 \\
18 \\
\downarrow \\
19\end{array}$} \\
\hline & 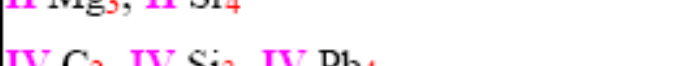 & 0 & 3 & 12 & \\
\hline 4 & 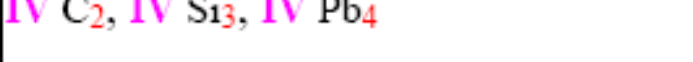 & 9 & 3 & 12 & \\
\hline 6 & $\mathrm{VI} \mathrm{O}_{3}, \mathrm{VI} \mathrm{S}_{4}$ & 7 & 2 & 12 & \\
\hline \multirow[t]{2}{*}{8} & \multirow[t]{2}{*}{ VIII $\mathrm{He}_{2}$, VIII $\mathrm{Ne}_{3}$, VIII Ar } & 14 & 4 & 32 & \\
\hline & & 37 & 11 & 60 & $50+10$ \\
\hline
\end{tabular}

Legend to Survey 9: The three end columns show significant numbers and their sums: the black numbers in the middle column represent the number of chemical elements in a given systemarrangement; to the left of them in red is the number of isotopes, and to the right, in pink, are the sums of the numbers denoting the groups in which the given elements are found. (All of this also applies to the next three Surveys.) 
Survey 10. "Element number", "Stable isotope number" and

"Groupe number" in $(\mathrm{d}, \mathrm{f})$ stable poly-isotopic elements

\begin{tabular}{|c|c|c|c|c|}
\hline 1 & $\mathrm{I} \mathrm{Cu}_{2}, \mathrm{I} \mathrm{Ag}_{2}$ & 4 & 2 & 2 \\
\hline 5 & $\mathrm{~V} \mathrm{Ta}_{2}$ & 2 & 1 & 5 \\
\hline \multirow[t]{2}{*}{9} & \multirow[t]{2}{*}{$\mathrm{IX} \mathrm{Ir}_{2}$} & 2 & 1 & 9 \\
\hline & & 08 & 4 & 16 \\
\hline 2 & II $\mathrm{Zn}_{5}$, II Hg & 19 & 3 & 6 \\
\hline 4 & IV Ti5, IV Ce 4 & 9 & 2 & 8 \\
\hline 6 & $\mathrm{VI} \mathrm{Cr} 4$ & 4 & 1 & 6 \\
\hline 8 & VIII $\mathrm{Fe}_{4}$, VIII $\mathrm{Ru}_{7}$ & 11 & 2 & 16 \\
\hline 10 & $\mathrm{X} \mathrm{N}_{5}, \mathrm{X} \mathrm{Pd}_{6}$ & 11 & 2 & 20 \\
\hline 12 & XII Dy7 & 7 & 1 & 12 \\
\hline 14 & $\mathrm{XIV} \mathrm{Er}_{6}$ & 6 & 1 & 14 \\
\hline & & 67 & 12 & 82 \\
\hline
\end{tabular}

Survey 11. "Element number", "Stable isotope number" and "Groupe number" in (s, p) stable monoisotopic elements

\begin{tabular}{|c|l|c|c|c|}
\hline 1 & $\mathrm{I} \mathrm{Na}_{1}, \mathrm{I} \mathrm{Cs}_{1}$ & 2 & 2 & 2 \\
3 & $\mathrm{III} \mathrm{Al}_{1}$ & 1 & 1 & 3 \\
5 & $\mathrm{~V} \mathrm{P}_{1}, \mathrm{~V} \mathrm{As}_{1}, \mathrm{~V} \mathrm{Bi}_{1}$ & 3 & 3 & 15 \\
7 & $\mathrm{VII} \mathrm{F}_{1}, \mathrm{VII} \mathrm{I}_{1}$ & 2 & 2 & 14 \\
& \multicolumn{2}{|c|}{$\mathbf{0 8}$} & $\mathbf{8}$ & $\mathbf{3 4}$ \\
\hline 2 & $\mathrm{II} \mathrm{Be}_{1}$ & 1 & 1 & $0 ?$ \\
\hline \multicolumn{4}{|c|}{$(8+1=9),(8+1=9),(34+0=34)$} \\
\hline
\end{tabular}


Survey 12. "Element number", "Stable isotope number" and

"Groupe number" in (s, p) stable monoisotopic elements

\begin{tabular}{|c|l|c|c|c|}
\hline 1 & $\mathrm{I} \mathrm{Au}_{1}, \mathrm{I} \mathrm{Tm}_{1}$ & 2 & 2 & 2 \\
3 & $\mathrm{III} \mathrm{Sc}_{1}, \mathrm{III} \mathrm{Y}_{1}$ & 2 & 2 & 6 \\
5 & $\mathrm{~V} \mathrm{Nb}_{1}, \mathrm{~V} \mathrm{Pr}_{1}$ & 2 & 2 & 10 \\
7 & $\mathrm{VII} \mathrm{Mn}_{1}$ & 1 & 1 & 7 \\
9 & $\mathrm{IX} \mathrm{Co}_{1}, \mathrm{IX} \mathrm{Rh}_{1}$ & 2 & 2 & 18 \\
11 & $\mathrm{XI} \mathrm{Tb}_{1}$ & 1 & 1 & 11 \\
13 & $\mathrm{XIII} \mathrm{Ho}_{1}$ & 1 & 1 & 13 \\
\hline \multicolumn{2}{|r|}{$(11),(11),(67)$} & $\mathbf{1 1}$ & 67 \\
\hline
\end{tabular}


Survey 13. "Element number", "Stable isotope number" and "Groupe number" in stable poly-isotopic and monoisotopic elements (I)

\begin{tabular}{l} 
Poly $(\mathrm{s}+\mathrm{p}):(18+37=55),(9+11=20),(41+60=101)$ \\
Poly $(\mathrm{d}+\mathrm{f}):(08+67=75),(4+12=16),(16+82=98)$ \\
\hline Total: $(55+75=\underline{\mathbf{1 3 0}}),(20+16=\underline{\mathbf{3 6}}),(101+98=\underline{\mathbf{1 9 9}})$ \\
\hline Mono $(\mathrm{s}+\mathrm{p}):(8+1=9),(8+1=9),(34+0=34)$ \\
Mono $(\mathrm{d}+\mathrm{f}): \quad(11), \quad(11), \quad(67)$ \\
Total: $(9+11=\underline{\mathbf{2 0}}),(9+11=\underline{\mathbf{2 0}}),(34+67=\underline{101})$ \\
\hline \\
Total sum: $(130+20=\underline{\mathbf{1 5 0}}),(36+20=\underline{\mathbf{5 6}}),(199+100=\underline{\underline{\mathbf{3 0 0}}})$ \\
\hline
\end{tabular}

Legend to Survey 13: The display of relationships in quantities shown in Surveys 9-12, by the key: poly-isotopy versus mono-isotopy.

Survey 14. "Element number", "Stable isotope number" and "Groupe number" in stable poly-isotopic and monoisotopic elements (II)

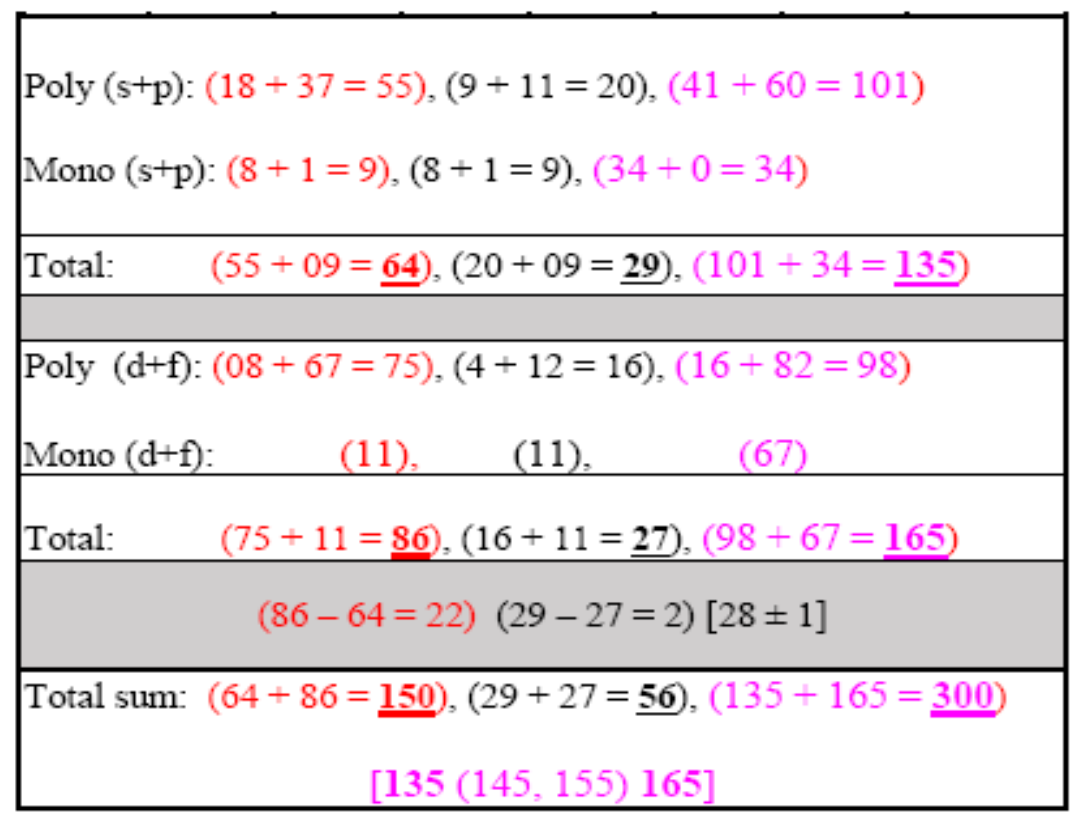

Legend to Survey 13: The display of relationships in quantities shown in Surveys 9-12, by the key: $(s+p)$ versus $(d+f)$ elements. 


\section{Additional materials}

Table A1. The Sukhodolets' system of amino acids in a 4 x 5 arrangement.

\begin{tabular}{|c|c|c|}
\hline The number of $\mathrm{H}$ atoms (in brackets) and nucleons & & \\
\hline \begin{tabular}{|c|c|c|c|c|c|}
$G(01) 01$ & $A(03) 15$ & $S(03) 31$ & $D(03) 59$ & $C(03) 47$
\end{tabular} & (13) 153 & \\
\hline 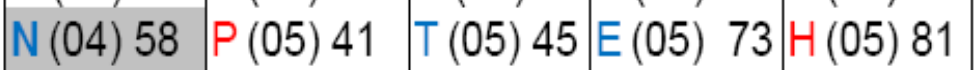 & (24) 298 & $(59 / 58)$ \\
\hline $\mathrm{F}(07) 91 \mathrm{M}(07) 75 \mathrm{Y}(07) 107$ & (34) 388 & $569 / 686$ \\
\hline $\mathrm{W}(08) 130 \mathrm{R}(10) 100 \mid \mathrm{K}(10) 72 \mathrm{I}(09) 57 \mathrm{~L}(09) 57$ & (46) 416 & \\
\hline $\begin{array}{l}569 \text { as neutron number and } 686 \text { as proton numbe } \\
569-59=627-117 \\
686-58=628\end{array}$ & & \\
\hline
\end{tabular}

Legend to Table A1: "The Sukhodolets' system of amino acids in a 4 x 5 arrangement (Sukhodolets, 1985): the system of 4 x 5 AAs. The shadow space: AAs with even number of hydrogen atoms $(4,6,8,10)$; the non-shadow space: AAs with odd number of hydrogen atoms $(1$, $3,7,9,11)$. In brackets: number of hydrogen atoms and out of brackets the number of nucleons. Nucleon number through a specific "simulation": 569 within two outer rows, as the number of neutrons, 569, in all 20 AAs - within their side chains; and 686 nucleons within two inner rows, as the number of protons, 686, in all 20 AAs - within their side chains. ... Within 20 side chains of amino acid molecules there are 569 neutrons as well as 569 non-hydrogen protons. Within 20 side chains of amino acid molecules there are 117 hydrogen protons, what means 117 hydrogen atoms at the same time $(117=59+58)$ " (MMR, 2011b, Table 7, p. 830). (Additional Note: In the original Sukhodolets' article, the number of nucleons is given in the whole amino acid molecule, but here only in the side chain.) 
Table A2. Atom number within 20 side chains of AAs as a quartet of even numbers

\begin{tabular}{|c|c|c|c|}
\hline out & in & out & in \\
\hline$G(01)$ & $\mathrm{N}(08)$ & $G(01)$ & $S(05)$ \\
\hline W (18) & $Q(11)$ & $\mathrm{A}(04)$ & $\mathrm{T}(08)$ \\
\hline $\mathrm{A}(04)$ & $S(05)$ & $L(13)$ & I (13) \\
\hline$C(05)$ & $\mathrm{D}(07)$ & $V(10)$ & $\mathrm{D}(07)$ \\
\hline$P(08)$ & $\mathrm{T}(08)$ & $P(08)$ & $E(10)$ \\
\hline $\mathrm{H}(11)$ & $E(10)$ & $R(17)$ & K (15) \\
\hline$V(10)$ & $F(14)$ & $Y(15)$ & $F(14)$ \\
\hline$Y(15)$ & $M(11)$ & W (18) & $Q(11)$ \\
\hline $\mathrm{R}(17)$ & $\mathrm{K}(15)$ & $\mathrm{H}(11)$ & $\mathrm{N}(08)$ \\
\hline$L(13)$ & I (13) & $C(05)$ & $M(11)$ \\
\hline $\begin{array}{ll}0 & 40 \\
F & 62\end{array}$ & 50 & 48 & 50 \\
\hline 102 & 102 & 102 & 102 \\
\hline
\end{tabular}

Legend to Table A2: "Atom number within 20 side chains of AAs as a quartet of even numbers. The outer/inner amino acid pairs which follow from ...Table A1. On the left: the original order as in Table A1; on the right: the chemical order of AAs as it is explained in Section 3.3" (MMR, 2011b, Table 9, p. 830). [Additional Note 1: The chemical order of AAs in the two right columns represents our view of arranging the two left Sukhodolets' columns according to the chemical similarity of amino acid molecules: to have the best possible chemical "alignment" according to chemical similarity in both readings - vertical and horizontal; examples of good chemical agreement: GA, AL, LV, VP, YW, WH; LI, RK, YF, CM; examples of less good agreement: PR, HC; GS, VD etc. Additional Note 2: Chemical similarity is not as well arranged here as it was in the arrangement: MMR, 2019a, Tab. 2, p. 14; because there AAs were viewed as free, independent of GC, and here is their order conditioned by the previous order of AAs determined by the number of hydrogen atoms in amino acid molecules. Additional Note 3: The sequence 4850-52-54 is given also in Eq. 5 in (MMR, 2011b, p. 829).] 
Table A3. The natural order of protein amino acids

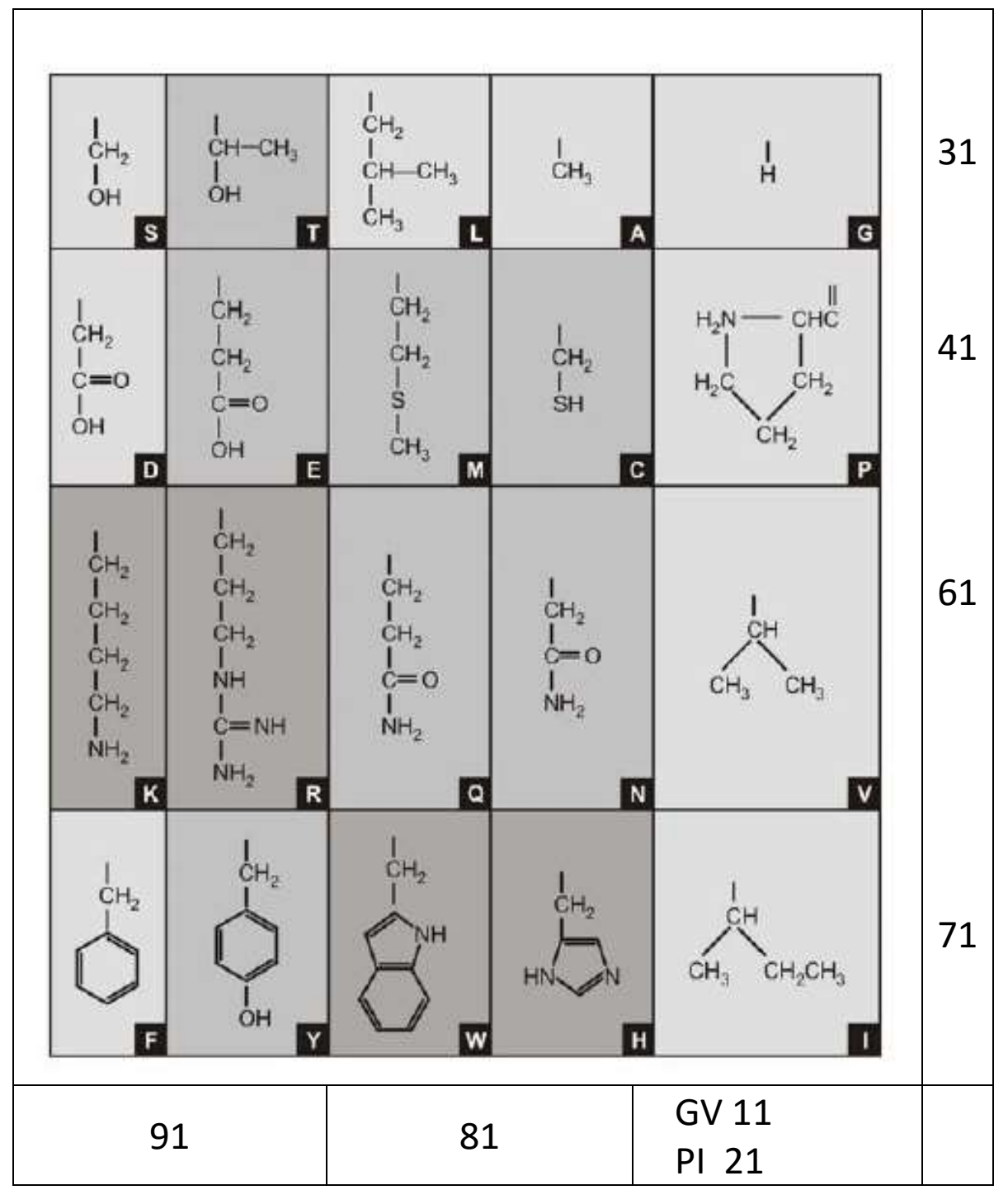

Legend to Table A3: The order of protein amino acids corresponding to the diagonal in the Periodic System of Numbers (Table A4): "The structure of amino acid molecules. The simplest amino acid is glycine $(\mathrm{G})$ whose side chain is only one atom of hydrogen. It is followed by alanine (A) whose side chain is only one $\mathrm{CH}_{3}$ group, which is the smallest hydrocarbon group. There are total of 16 amino acids of alanine stereochemical type ... with one $\mathrm{CH}_{2}$ group, each between the "body" and the "head". The glycine type contains glycine (G) only; valine type contains valine and isoleucine $(\mathrm{V}, \mathrm{I})$. The last stereochemical type is proline type with proline $(\mathrm{P})$ which represents the inversion of valine in the sense that the "triangle" of three $\mathrm{CH}_{2}$ groups for the "head" is not bound by the basis, therefore not only with one but with two $\mathrm{CH}_{2}$ groups (Popov, 1989; Rakočević \& Jokić, 1996). Light tones [(G, P, V, I) (32 atoms)] \& [(A, L, S, D, F) (43 atoms)]: invariant AAs; most dark tones [(K, R, W, H) (61 atoms]: most variant AAs; less dark tones [(T, E, M, C, Q, N, Y) (68 atoms)]: less variant AAs. Notice a further possibility for splitting: 1. two aliphatic AAs [(K, R) (32 atoms)], 2. two aromatic AAs [(W, H) (29 atoms)], 3. chalcogene AAs plus aromatic hydroxide derivative [(MC, TY) (39 atoms), and 4. dicarboxylic 
amino acid plus two amides [(E, QN) (29 atoms). A specific calculation: less variant AAs, all aliphatic but one (Y), plus two more variant (aromatic: W, H), equals $68+29=97$ atoms; invariant AAs, all aliphatic but one (F) plus two more variant (aliphatic: K, R), equals $75+32=$ 107 atoms; if so, then: $107-97=10 ; 107+97=204 \ldots$... [ Additional Note: Generating the arrangement of AAs follows from the natural order of AAs given in Tables A5 and A6. The logic of classifications and distinctions, given by shading, is explained in Table A7.]

Table A4. The periodic system of numbers (PSN) in decimal number System

\begin{tabular}{|c|c|c|c|c|c|c|c|c|c|c|c|}
\hline$(-2)$ & $\begin{array}{l}\cdots \\
\cdots\end{array}$ & & & & & & & & & $\ldots$ & -22 \\
\hline$(-1)$ & -21 & -20 & -19 & -18 & -17 & -16 & -15 & -14 & -13 & -12 & -11 \\
\hline$(0)$ & -10 & -09 & -08 & -07 & -06 & -05 & -04 & -03 & -02 & -01 & 00 \\
\hline (1) & 01 & 02 & 03 & 04 & 05 & 06 & 07 & 08 & 09 & 10 & 11 \\
\hline (2) & 12 & 13 & 14 & 15 & $16^{-}$ & $-47^{-}$ & -18 & 19 & 20 & 21 & 22 \\
\hline (3) & 23 & 24 & 25 & $26^{\prime}$ & 27 & 28 & 29 & 30 & 31 & 32 & 33 \\
\hline (4) & 34 & 35 & 36 & 37 & 38 & 39 & 40 & 41 & 42 & 43 & 44 \\
\hline (5) & 45 & 46 & 47 & 48 & 49 & 50 & $\underline{51}$ & 52 & 53 & 54 & 55 \\
\hline (6) & 56 & 57 & 58 & 59 & 60 & 61 & 62 & 63 & 64 & 65 & 66 \\
\hline (7) & 67 & 68 & 69 & 70 & 71 & 72 & 73 & 74 & 75 & 76 & 77 \\
\hline (8) & 78 & 79 & 80 & 81 & 82 & 83 & 84 & 85 & 86 & 87 & 88 \\
\hline (9) & 89 & 90 & 91 & 92 & 93 & 94 & 95 & 96 & 97 & 98 & 99 \\
\hline (A) & $\mathrm{AO}$ & A1 & $\mathrm{A} 2$ & A3 & A4 & A5 & A6 & A7 & A8 & A9 & $\mathrm{AA}$ \\
\hline (B) & B1 & B2 & B3 & B4 & B5 & B6 & B7 & B8 & $\mathrm{B} 9$ & BA & $\mathrm{BB}$ \\
\hline
\end{tabular}

Legend to Table A4: Periodic System of numbers in decimal number system as in MMR, 2011b, Tab. 4, p. 826 and in MMR, 2019a, Table A1, p. 28. 
Table A5. The atom number within 8 pairs of alanine stereochemical type of AAs.

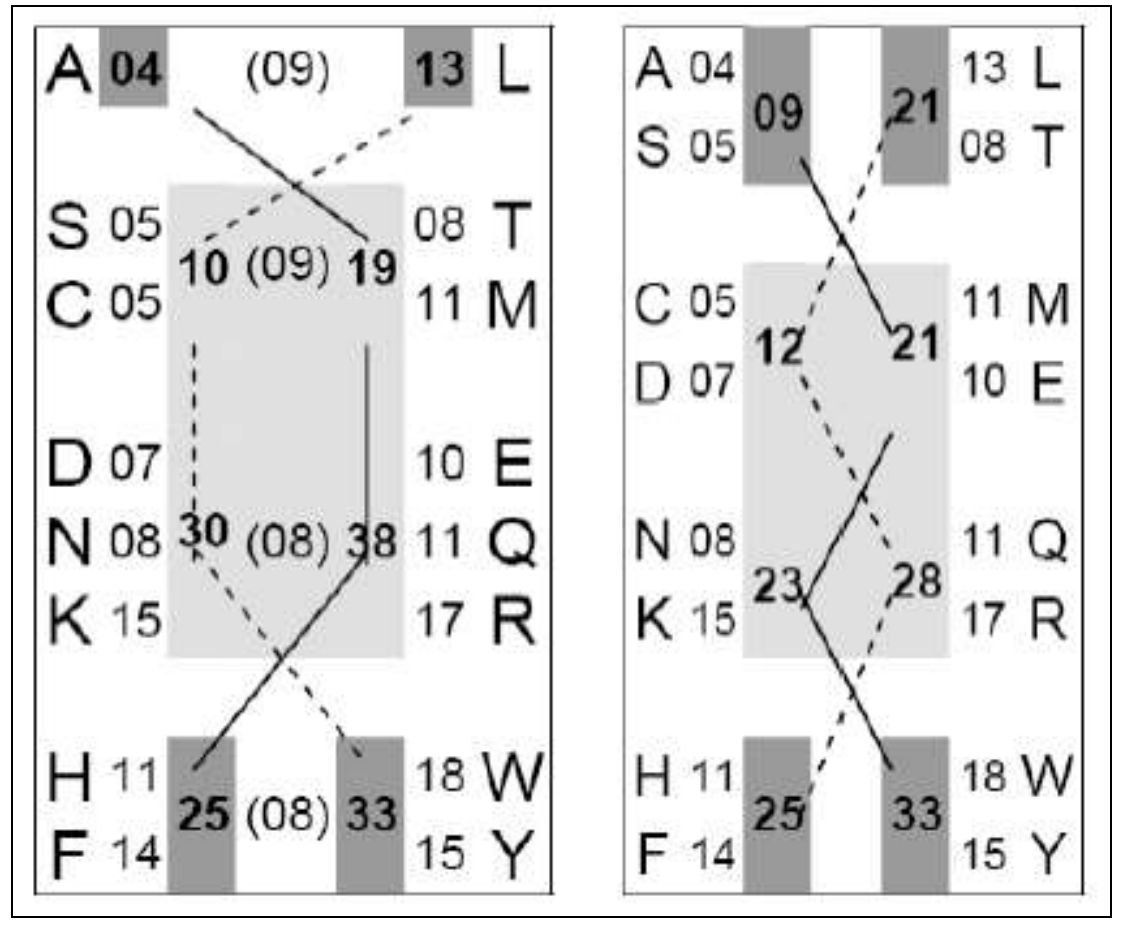

Legend to Table A5: The excerpt from original text in MMR, 2011b, Section 2, Table 2.1, p. 823): "The atom number within 8 pairs of alanine stereochemical type of AAs. On the full line, as well as on the dotted one, there are 86 atoms; the differences 8 and $9(9-8=1)$ express "the minimum change relation among the amino acids" (Swanson, 1984, p 191). The order follows from the atom number hierarchy ..." [Additional Note: Amino acids are read here according to the Gray code model, that is, following the edges of Boolean logic square: STLA, DEMC, etc. and so as we find them in Table 3. On the other hand, in the order of AAs according to chemical similarity (MMR, 2019a, Tab. 2 in relation to Tab. 3), AAs go through the diagonals of the logical square.] 
Table A6 (on the left): The atom number within four pairs of non-alanine sterepchemical types of AAs

Table A7 (on the right): The variability of AAs with respect to their polarity

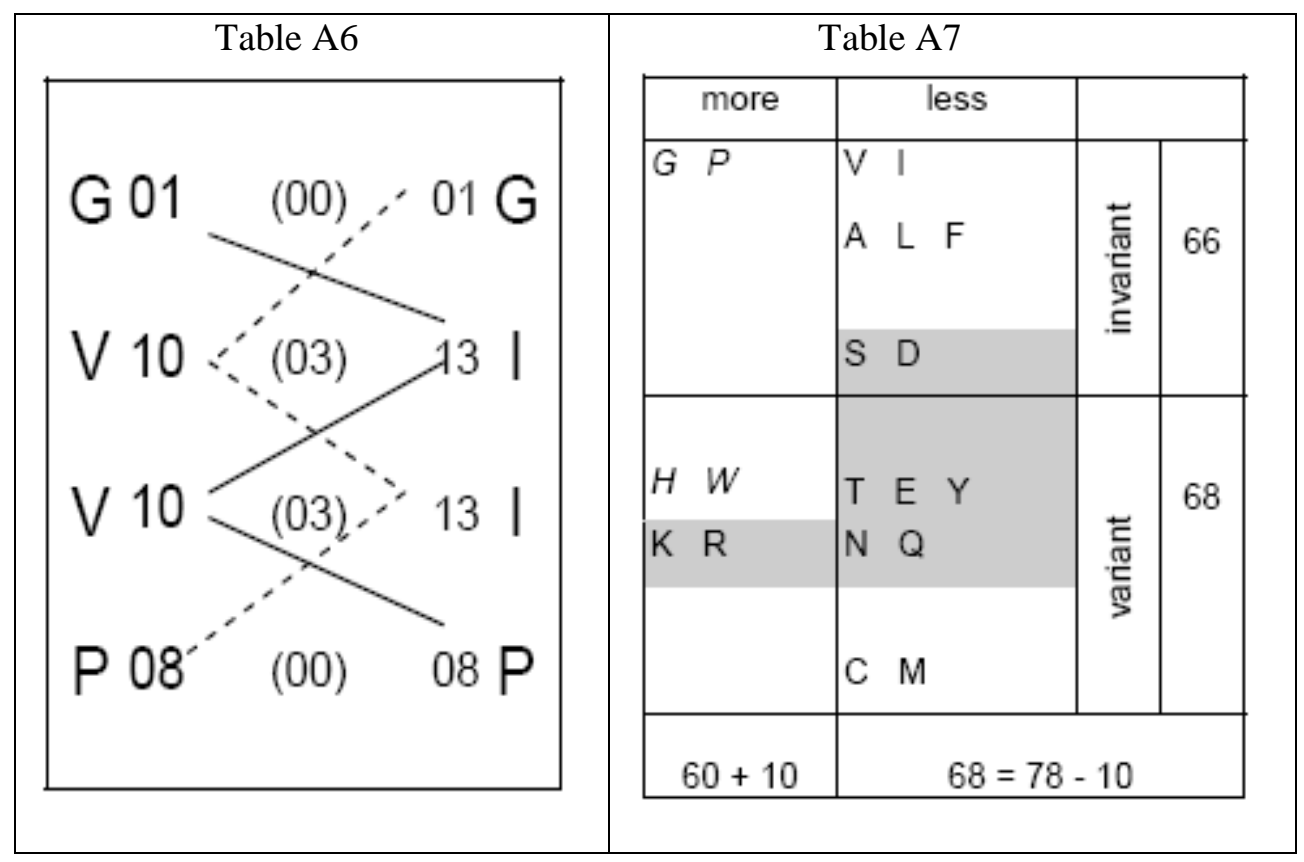

Legend to Table A6: "The atom number within four pairs of non-alanine types of AAs. The two lines have 32 atoms each. The number of amino acids must be doubled through the pairs G-G and P-P; the amino acid order comes from the atom number hierarchy" (MMR, 2011b, Tab. 2.2, p. $823)$.

Legend to Table A7: "The variability of AAs with respect to their polarity ..." (MMR, 2011b, Fig. 11, p. 835) (See Explanation of Table A7 in Box 4.) 
Table A8. The source of mirror symmetry within Protein Amino Acid System (PAAS)

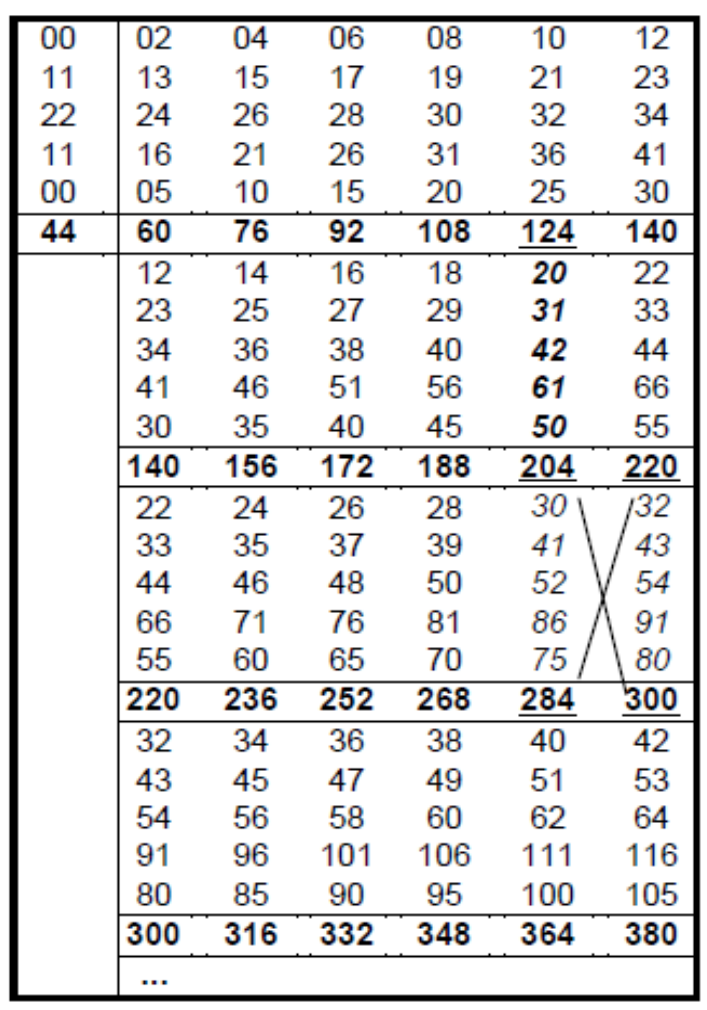

Legend to Table A8. "The arrangement represents the Table of Distinct 2-5 Adding (TDA) with starting column 00-11-22-11-00 which follows from PSN (Periodic system of numbers: Table A4) in decimal number system by overlapping the real sequence of doubled the first possible triangle in Boolean space (0-1-2) with its mirror image through compression and superposition at the point "22"). In the $10^{\text {th }}$ step we have a realization of the sequence (20-31-42-61-50), the same with the number of atoms in five AAs classes $(20,31,42,61,50)$ as it is here presented: all five results in the 10th step are mirror image of the first step." 
Table A9. The order of five quartets of protein amino acids following from Table A8

\begin{tabular}{|cccccc|}
\hline \multicolumn{7}{|c|}{$[72(78-6)]$} & {$[12 \times 6]$} \\
$G(01)$ & $\mathrm{A}(04)$ & $\mathrm{N}(08)$ & $\mathrm{D}(07)$ & $\rightarrow$ & 20 \\
$\mathrm{~V}(10)$ & $\mathrm{P}(08)$ & $\mathrm{S}(05)$ & $\mathrm{T}(08)$ & $\rightarrow$ & 31 \\
$\mathrm{I}(13)$ & $\mathrm{L}(13)$ & $\mathrm{C}(05)$ & $\mathrm{M}(11)$ & $\rightarrow$ & 42 \\
$\mathrm{~K}(15)$ & $\mathrm{R}(17)$ & $\mathrm{F}(14)$ & $\mathrm{Y}(15)$ & $\rightarrow$ & 61 \\
$\mathrm{Q}(11)$ & $\mathrm{E}(10)$ & $\mathrm{W}(18)$ & $\mathrm{H}(11)$ & $\rightarrow$ & 50 \\
$51-1$ & $51+1$ & $51-1$ & $51+1$ & \\
\hline \multicolumn{7}{|c}{} \\
\hline $132(2 \times 66)]$ & {$[22 \times 6]$} \\
\hline
\end{tabular}

Legend to Table A9: "This Table follows from Table A8 and PSN (Table A4), from a doubled starting triangle from the top of the last column; triangle switched with its mirror image and superimposed: (00-11-22 / 22-11-00 $\rightarrow$ 00-11-22-11-00 ...” (MMR, 2019a, Table 3).

Table A10. The determinations on the six-bit binary tree

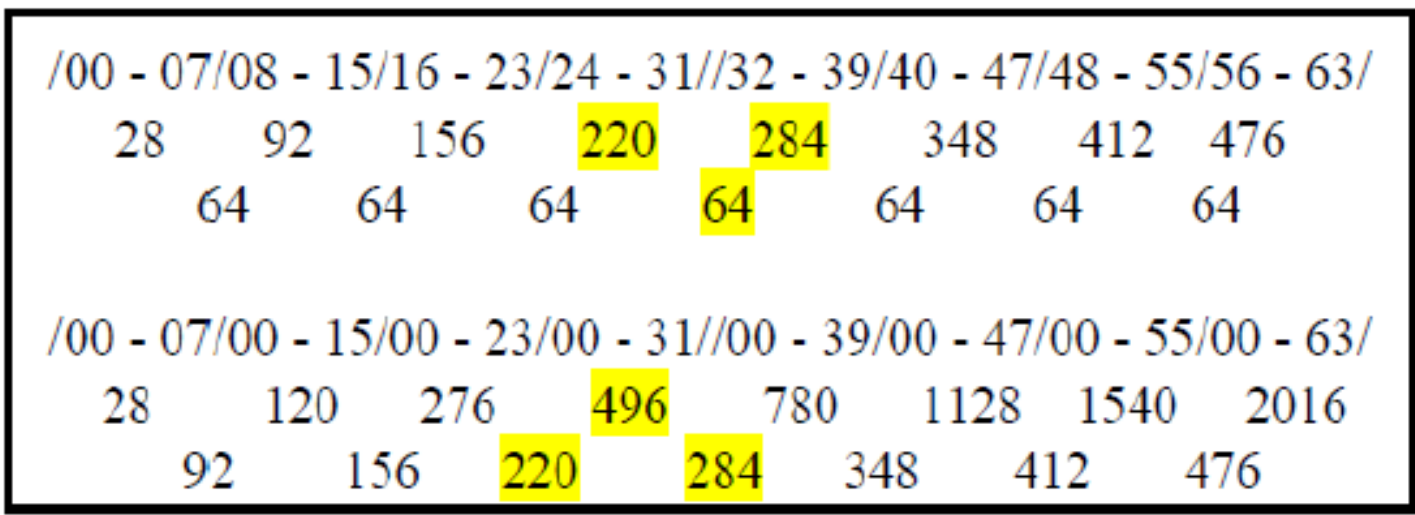

Legend to Table A10: The Table follows from (MMR, 1997b, Figure 7, p. 60): "The determination of the series of the numbers $0-63$. When we look closely into the structure of the sequence $0-63$ of the series of the natural numbers we come to the obvious and self-evident explanation of the reason why the genetic code must be six-bit code, no matter if it is the manifestation in the form of the Gray Code model (Swanson, 1984, p 188), or it is in the form of the Binary tree (Rakočević, 1998, p 284). There must be 8 codons, i.e. amino acid classes. The structure of the sequence $0-63$ is strictly determined by third perfect number (496) and the sum consisted of the first pair of the friendly numbers $(220+284)$. Along with this, the specific Boolean square is being made and it is the restrictive factor, in a sense that it is not possible to ' $\mathrm{go}$ on' any further - not ahead, not back: (0) $220+284=504$; (1) $156+348=504$; (2) $92+412=504$; (3) $28+476=504$. The key distinctions within the genetic code are obviously self-evident: entity 64 as a series of continuity (correspondent with 64 codons); entity 20 from a difference $496-476=20$ (correspondent with 20 amino acids) (496, the third perfect number) etc." (Additional Note: The Table was republished in: MMR, 2019a, p. 35.) 


\section{Backup materials}

Backup materials contain information about facts that are, or could be in a relationship, that is, in correspondence with the chemical and/or genetic code.

Table B1. The uniqueness of numbers 89

\section{9, 109 and the Fibonacci Sequence}

May 15,2012 by Gary Meisner 16 Comments

The reciprocal of 89 , a Fibonacci number, is based on the Fibonacci series.

This is a little curiousity involving the number 89 , one of the Fibonacci series numbers.

$1 / 89$ is a repeating decimal fraction with 44 characters:

.01123595505617977528089887640449438202247191

You can see the beginning of the Fibonacci sequence in the first 6 digits of the decimal equivalent of $1 / 89$. (i.e., $0,1,1,2,3,5$ appears as 0.011235 ..)

Table B2. The uniqueness of number 109

\section{The reciprocal of 109 is also based on the Fibonacci series, forwards and backwards}

Here's another curiousity involving the number 109 , discovered and contributed (10/20/2003) by Rick Toews.

$1 / 109$ is a repeating decimal fraction with 108 characters:

.009174311926605504587155963302752293577981651376146788 990825688073394495412844036697247706422018348623853211

You can see the beginning of the Fibonacci sequence in the LAST 6 digits of the decimal equivalent of $1 / 109$, appearing in REVERSE order starting from the END of the decimal. (i.e., $0,1,1,2,3,5,8$ appears as ...853211)

https://www.goldennumber.net/89-and-109/ 
Table B3. The arithmetical source of atom number in 61 amino acids (within their side chains)

\begin{tabular}{|cccccccc|c|}
\hline 28 & 92 & 156 & 220 & 284 & 348 & 412 & 476 & \\
144 & 96 & 48 & & & 48 & 96 & 144 & 504 \\
172 & 188 & 204 & 220 & 284 & 300 & 316 & 332 & $\times 16$ \\
200 & 280 & 360 & 440 & 568 & 648 & 728 & 808 & - \\
& & & & & & & & 8064 \\
$(200+808=504 \times 2)+(280+728=504 \times 2)$ & $\ldots$ & $\rightarrow 504 \times 8$ & \\
44 & 60 & 76 & 92 & 108 & 124 & 140 & 156 & 8658 \\
304 & 304 & 304 & 304 & 304 & 304 & 304 & 304 & 8064 \\
348 & 364 & 380 & 396 & 412 & 428 & 444 & 460 & 594 \\
392 & 424 & 456 & 488 & 520 & 552 & 584 & 616 & \\
$(392+616=504 \times 2)+(424+584=504 \times 2) \ldots$ & $\rightarrow 504 \times 8$ & \\
\hline
\end{tabular}

Legend to Table B3: The table follows from the merging of two arithmetic arrangements, presented in Table A8 and A10, respectively. [Above: from Tab. 10 are taken 28-476; from Tab. 8: 172-332; Down: from Tab. 8: 44-156 and 348-460.] The total sum of the numbers in front of and behind the logical square: [(0) 204, (1) 220, (2) 284, (3) 300] is 8064, which is 594 less than the sum of the first four perfect numbers, and that is exactly the number of atoms in 61 amino acid molecules (cf. Table 9). The following regularity can be noticed here. There are four quantities left and right (including two members of the first friendly pair of numbers (220-284). The distances left and right of the friendly pair are: 64 in the first and 16 in the second row. Number 4 corresponds to 2-bit, number 16 4-bit and number 64 6-bit binary tree. That is analogous to the situation we have in Tables 7 and 8.

Table B4. The arithmetical source of atom number in 20 amino acids (within their whole molecules)

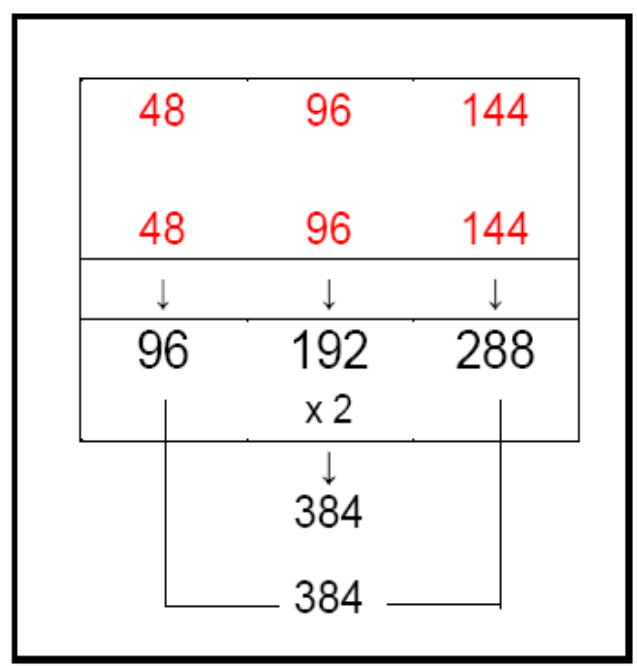

Legend to Tab. B4: The result follows from the differences between 
first two rows in Tab. B3.

Table B5. The arithmetical source of the number of stable chemical elements

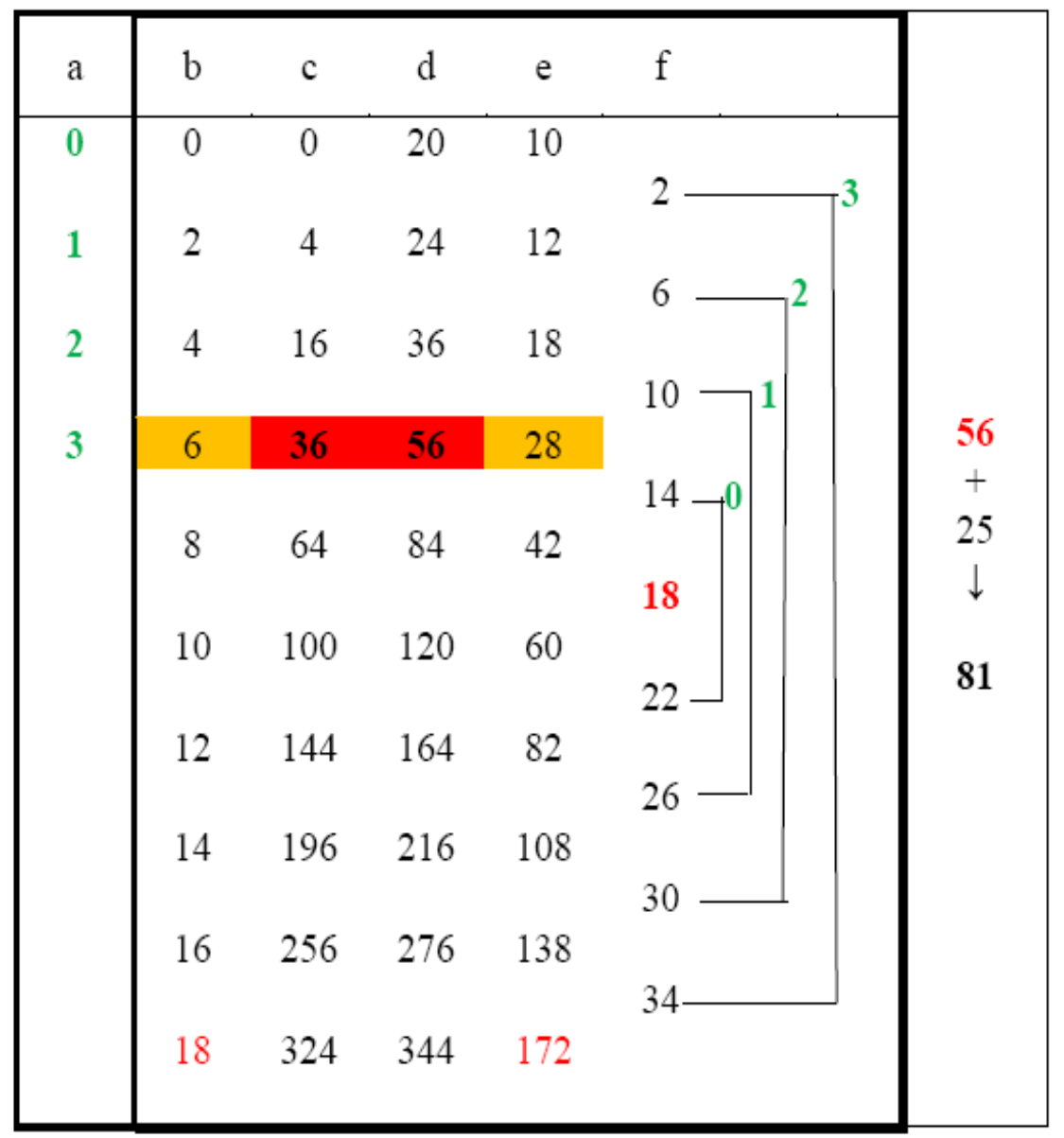

Legend to Tab. B5: Number of stable elements with a unique position in a unique arithmetic system: a. ordinal number; b. a sequence of even natural numbers generated in Boolean spaces; $c$. squares of numbers from column "b"; d. numbers in column "c" increased by 20 ; e. halves of the numbers in column "d"; f. differences every two numbers in column "e". The sum of the stable and unstable elements is given in the far right column: a total of 81 up to the stability / instability limit in PSE, up to Polonium. The idea of the hypothesis of the existence of a logical square within a unique arithmetic arrangement is indicated in green. Notice that the connection with the arithmetic arrangement in Table B3 is established via the limit number 172: here it is the last number, and there it is the first. 
Table B6. The arithmetical source of the number of monoisotopic chemical elements

\begin{tabular}{|c|c|c|c|c|c|c|c|c|c|c|c|c|}
\hline (1) & 1 & + & 9 & $=$ & 10 & 4 & + & 1 & $=$ & 05 & (1) & \\
\hline (2) & 2 & + & 9 & $=$ & 11 & 04 & + & 2 & $=$ & 06 & $(2)$ & \\
\hline (3) & 3 & + & 9 & $=$ & 12 & 4 & + & 3 & $=$ & 07 & (3) & 59 \\
\hline (4) & 4 & + & 9 & $=$ & 13 & 4 & + & 4 & $=$ & 08 & (4) & 101 \\
\hline ( & 5 & + & 9 & $=$ & 14 & 4 & + & 5 & $=$ & 09 & (5) & \\
\hline (6) & 6 & + & 9 & $=$ & 15 & 4 & + & 6 & $=$ & 10 & (6) & \\
\hline (7) & 7 & + & 9 & $=$ & 16 & 4 & + & 7 & $=$ & 11 & (7) & $010(2)$ \\
\hline (8) & 8 & + & 9 & $=$ & 17 & 4 & + & 8 & $=$ & 12 & (8) & $101(5)$ \\
\hline (9) & 9 & + & 9 & $=$ & 18 & 4 & + & 9 & $=$ & 13 & (9) & \\
\hline
\end{tabular}

Explanation in Box 3. 
Table B7. The multiples of perfect numbers

\begin{tabular}{|c|c|c|c|}
\hline 6 & 28 & 496 & 8128 \\
\hline 1 & 1 & 1 & 1 \\
$2-0$ & 2 & 2 & 2 \\
$4-1$ & $4-0$ & 4 & 4 \\
\hline $8-2$ & $8-1$ & 8 & 8 \\
\hline & $16-2$ & $16-0$ & 16 \\
& $32-4$ & $32-1$ & $32-2$ \\
& $64-2$ & $64-0$ \\
& & $128-4$ & $128-1$ \\
& & $256-8$ & $256-2$ \\
& & $512-16$ & $512-4$ \\
& & $1024-8$ \\
& & $2048-16$ \\
& & $4096-32$ \\
& & $8192-64$ \\
\hline
\end{tabular}


Table B8. The multiples of the third friendly number

\begin{tabular}{|c|c|c|}
\hline 1 & 1 & 1 \\
2 & 2 & 2 \\
4 & 4 & 4 \\
8 & 8 & 8 \\
16 & 16 & 16 \\
$32+00$ & $32+5 \times 0$ & 32 \\
$32+05$ & $32+5 \times 1$ & 37 \\
$64+10$ & $64+5 \times 2$ & 34 \\
$128+20$ & $128+5 \times 4$ & 148 \\
$256+40$ & $256+5 \times 8$ & 296 \\
$512+80$ & $512+5 \times 16$ & 592 \\
\hline $124+160$ & $1024+5 \times 32$ & 1184 \\
\hline
\end{tabular}


Table B9. The logic of perfect numbers

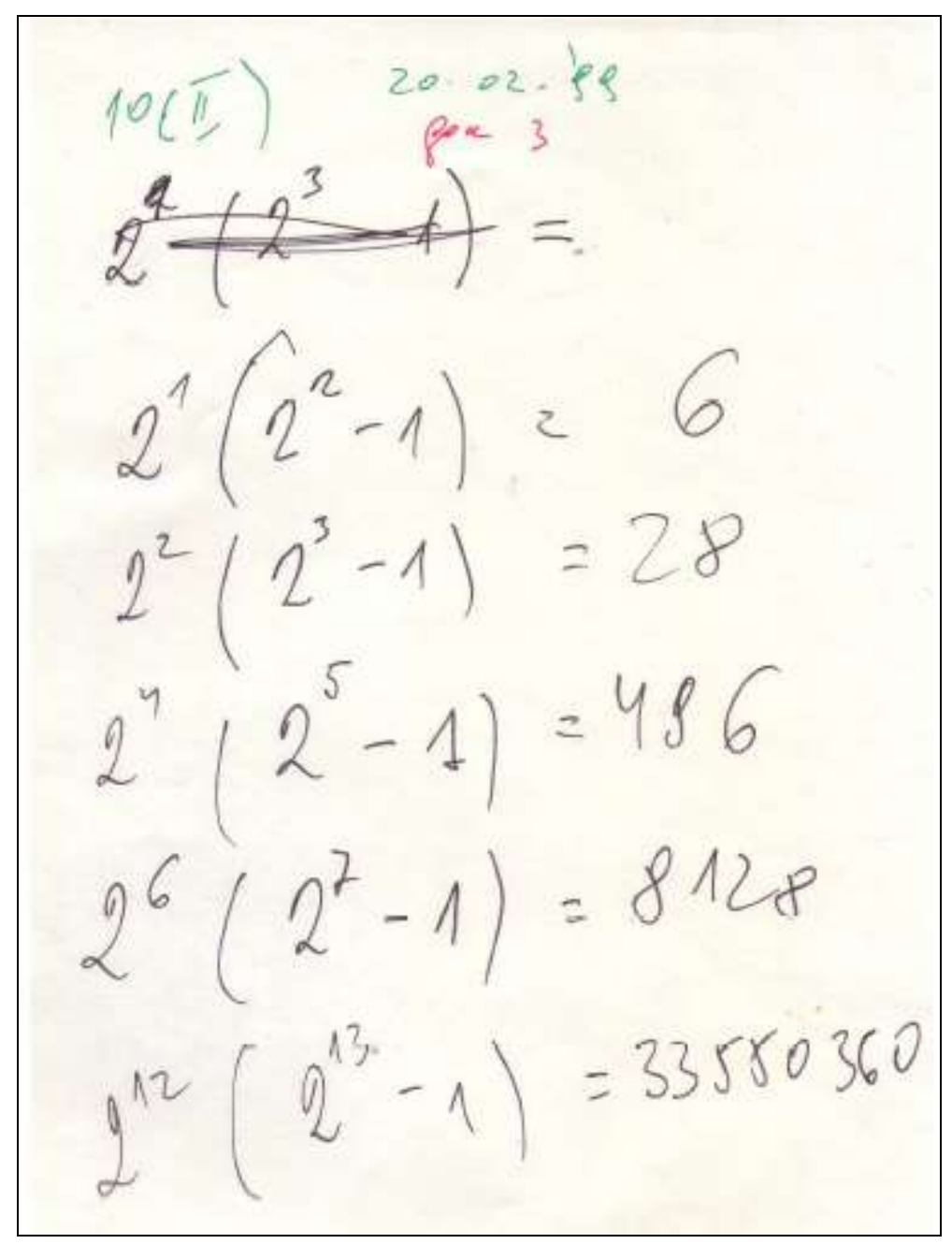

56 